\title{
ON SOME PERIODIC HARTREE-TYPE MODELS FOR CRYSTALS
}

\author{
I. CATTO ${ }^{\text {a }}$, C. LE BRIS ${ }^{\text {b }}$, P.-L. LIONS ${ }^{\text {a }}$ \\ ${ }^{a}$ CEREMADE, CNRS UMR 7534, Université Paris-Dauphine, Place de Lattre de Tassigny, \\ 75775 Paris cedex 16, France \\ ${ }^{\mathrm{b}}$ CERMICS, Ecole Nationale des Ponts et Chaussées, 6 \& 8, avenue Blaise Pascal, Cité Descartes, \\ Champs sur Marne, 77455 Marne-La-Vallée cedex, France
}

Received 28 September 2000

ABSTRACT. - We continue here our study of the thermodynamic limit for various models of Quantum Chemistry. More specifically, we study the Hartree and the restricted Hartree model. For the restricted Hartree model, we prove the existence of the thermodynamic limit for the energy per unit volume. We also define a periodic problem associated to the Hartree model, and we prove that it is well-posed.

(C) 2002 L'Association Publications de l'Institut Henri Poincaré. Published by Elsevier B.V. All rights reserved

RÉSUMÉ. - Nous poursuivons dans cet article notre étude systématique de la limite thermodynamique de divers modèles issus de la Chimie Quantique Moléculaire. Nous étudions plus spécifiquement les modèles de Hartree et de Hartree restreint. Pour le modèle de Hartree restreint, nous prouvons l'existence de la limite thermodynamique de l'énergie par unité de volume. Nous définissons également un modèle périodique associé au modèle de Hartree, et nous démontrons qu'il est bien posé.

(C) 2002 L'Association Publications de l'Institut Henri Poincaré. Published by Elsevier B.V. All rights reserved

\section{Introduction}

We consider here the thermodynamic limit (or bulk limit) problem for some Hartree type models, thereby continuing a long term work that we have begun in [11] with a similar study in the setting of the Thomas-Fermi-von Weizsäcker type models. The results we have obtained in that framework were summarized in [10], those we shall obtain here have been announced in [12]. It is to be mentioned that we also consider in [13] the same problem for the reduced Hartree-Fock and the Hartree-Fock models. For the sake of consistency, we briefly recall now the motivations of our work. We also say a few words on how this work interacts with other mathematical studies. And we refer the reader to [11] for a more detailed introduction.

The present work, as well as our previous ones, finds its roots in many mathematical studies devoted to the mathematical counterpart of problems of Statistical Mechanics.

E-mail address: catto@ceremade.dauphine.fr (I. Catto). 
Briefly speaking, the so-called thermodynamic limit problem consists of examining the behaviour of models for a finite volume of matter when the volume under consideration goes to infinity. Since the energy is an extensive thermodynamic quantity, it is expected that the energy per unit volume goes to a finite limit when the volume goes to infinity. It is also expected that the function representing the state of the matter goes also to a limit in some sense. To fix the ideas, let us make precise these questions in the case of an infinite crystal and in the setting of a model of the density functional theory. We shall see extensions of this simplified setting later on.

Consider a finite number of nuclei, each nucleus being of unit charge and being located at a point $k=\left(k_{1}, k_{2}, k_{3}\right)$ of integral coordinates in $\mathbf{R}^{3}$, which is the center of a cubic unit cell $Q_{k}=\left\{\left(x_{1}, x_{2}, x_{3}\right) \in \mathbf{R}^{3} ;-\frac{1}{2}<x_{i}-k_{i} \leqslant \frac{1}{2}, i=1,2,3\right\}$ (with the convention that $Q_{0}$ will be henceforth denoted by $Q)$. The set of the positions of these nuclei is then a finite subset $\Lambda$ of the set of all points of integral coordinates that is $\mathbf{Z}^{3} \subset \mathbf{R}^{3}$. The union of all cubic cells whose center is a point of $\Lambda$ is denoted by $\Gamma(\Lambda)$; its volume is denoted by $|\Lambda|$. Since each cell has unit volume and each nucleus is of unit charge, $|\Lambda|$ is also the number of nuclei and the total nuclear charge.

Suppose that for $\Lambda \subset \mathbf{Z}^{3}$ fixed, we have a well-posed model for the ground state of the neutral molecule consisting of $|\Lambda|$ electrons and $|\Lambda|$ nuclei located at the points of $\Lambda$. Let us denote by $I_{\Lambda}$ the ground-state energy, and by $\rho_{\Lambda}$ the minimizing electronic density.

Then, the question of the existence of the thermodynamic (or bulk) limit for the model under consideration may be stated as follows:

(i) Does there exist a limit for the energy per unit volume $\frac{1}{|\Lambda|} I_{\Lambda}$ when $|\Lambda|$ goes to infinity?

(ii) Does the minimizing density $\rho_{\Lambda}$ approach a limit $\rho_{\infty}$ (in a sense to be made precise later) when $|\Lambda|$ goes to infinity?

(iii) Does the limit density $\rho_{\infty}$ have the same periodicity as the assumed periodicity of the nuclei?

We shall not deal here with the physical background of this theoretical problem, and we refer the reader to the textbooks [4,51] and articles [26,30]. We prefer to concentrate ourselves on the mathematical works that are devoted to this difficult question.

The models we shall consider are models arising in Quantum Chemistry, and therefore models that are only valid at zero temperature. From the mathematical viewpoint, the thermodynamic limit problem has been extensively studied, in the zero temperature case as well as in the case of strictly positive temperatures.

A brief historical survey should go as follows. The story has really begun with Fischer and Ruelle, who have proved the existence of the thermodynamic limit for the (classical or quantum) microcanonical, canonical, and grand canonical ensembles for a system of particles in $\mathbf{R}^{d}$ (see [44], and references therein). It is worth noticing that their proof did not cover the case of a long range interaction like the Coulomb interaction. It is only in the late sixties that Lieb and Lebowitz, using a result by Dyson and Lenard, proved the existence of the thermodynamic limit for real matter, i.e. with Coulomb forces (see [25-27,24]). This undoubtedly constitutes the first milestone of the mathematical understanding of these problems of Statistical Mechanics. The proof has next been extended by Lieb and Narnhofer [31] in 1974 to deal with the case of Jellium, that is 
to say to deal with a model where the electrons are immersed in a uniformly positively charged background.

In 1985, Fefferman laid the second milestone by proving in [17] the existence of the thermodynamic limit for a crystal, in the statistical setting. For the first time, a statistical model of a non spherically symmetric matter was treated in this respect. With slight modifications, Fefferman's proof has been extended by Gregg in 1989 ([18]) to treat Coulomb-like interactions. Let us emphasize that the two main difficulties that we have just identified, namely the long range nature of the Coulomb potential and the (obvious) lack of spherical symmetry of the periodic lattices, will be of course also present in our work.

In this very brief survey, we have on purpose omitted to mention the ground-breaking work [32] by Lieb and Simon on the thermodynamic limit in the framework of the Thomas-Fermi theory (TF Theory for short). Indeed, this work is at the origin of our own study [11] on the Thomas-Fermi-von Weizsäcker model (TFW model for short), and has therefore a far larger impact on our work than the, however fundamental, works that we have quoted above.

At this stage of our short presentation of the state of the art of the mathematical knowledge on thermodynamic limit problems, we find it useful to briefly recall now the results that we have obtained in [11] on the Thomas-Fermi-von Weizsäcker model. Indeed, many of the concepts and techniques that we have used in [11] (some of them being inherited from Lieb and Simon, some others being especially introduced by us in order to treat the TFW case) will be useful here. Moreover, recalling the complete results we have obtained in the TFW case will help the reader to place the results we shall obtain here on the Hartree model in this context. It is also to be remarked that our results on the TFW model include Lieb and Simon results on the TF model (suppress simply the gradient term in the energy functional and make the quite obvious corresponding modifications in the sequel).

The Thomas-Fermi-von Weizsäcker model for the neutral molecular system described above is an improved form of the standard Thomas-Fermi model, and reads as follows

$$
\begin{aligned}
I_{\Lambda}^{T F W}=\inf \left\{E_{\Lambda}^{T F W}(\rho)\right. & \left.+\frac{1}{2} \sum_{y \neq z \in \Lambda} \frac{1}{|y-z|} ; \rho \geqslant 0, \sqrt{\rho} \in H^{1}\left(\mathbf{R}^{3}\right), \int_{\mathbf{R}^{3}} \rho=|\Lambda|\right\}, \\
E_{\Lambda}^{T F W}(\rho)= & \int_{\mathbf{R}^{3}}|\nabla \sqrt{\rho}|^{2}+\int_{\mathbf{R}^{3}} \rho^{5 / 3}-\int \sum_{\mathbf{R}^{3}}\left(\sum_{k \in \Lambda} \frac{1}{|x-k|}\right) \rho(x) \mathrm{d} x \\
& +\frac{1}{2} \iint_{\mathbf{R}^{3} \times \mathbf{R}^{3}} \frac{\rho(x) \rho(y)}{|x-y|} \mathrm{d} x \mathrm{~d} y .
\end{aligned}
$$

The TFW (and as well as the TF) model belongs to a large class of models that is today identified as the models arising in Density Functional Theory: we refer the reader to $[14,41]$ for an introduction to the general features and the physical foundations of such models. Mathematically, it is a well-known fact that the problem (1)-(2) has a unique 
minimizing density, denoted by $\rho_{\Lambda}$ (see Lieb [29], Benguria et al. [5], or Lions [35]), and that, denoting $u_{\Lambda}=\sqrt{\rho}_{\Lambda}, u_{\Lambda}$ is a solution to

$$
-\Delta u_{\Lambda}+\left[\frac{5}{3} \rho_{\Lambda}^{2 / 3}-\Phi_{\Lambda}\right] u_{\Lambda}=-\theta_{\Lambda} u_{\Lambda},
$$

where we denote by

$$
\Phi_{\Lambda}=\sum_{k \in \Lambda} \frac{1}{|x-k|}-\rho_{\Lambda} \star \frac{1}{|x|},
$$

the effective potential the electrons experience, and where $\theta_{\Lambda}>0$ is the Lagrange multiplier associated to the constraint in (1).

In our previous work [11], we have proved that the three questions (i)-(ii)-(iii) of the thermodynamic limit problem that we have asked above can be answered positively in the setting of the TFW theory. More precisely, let us first of all introduce the periodic potential $G$ uniquely defined by

$$
-\Delta G=4 \pi\left(-1+\sum_{y \in \mathbf{Z}^{3}} \delta(\cdot-y)\right),
$$

and

$$
\int_{Q} G=0,
$$

and then define the following periodic minimization problem set on the unit cell $Q$ of the lattice

$$
\begin{gathered}
I_{p e r}^{T F W}=\inf \left\{E_{p e r}^{T F W}(\rho) ; \rho \geqslant 0, \sqrt{\rho} \in H_{p e r}^{1}(Q), \int_{Q} \rho=1\right\}, \\
E_{p e r}^{T F W}(\rho)=\int_{Q}|\nabla \sqrt{\rho}|^{2}+\int_{Q} \rho^{5 / 3}-\int_{Q} \rho(x) G(x) \mathrm{d} x \\
+\frac{1}{2} \iint_{Q \times Q} \rho(x) \rho(y) G(x-y) \mathrm{d} x \mathrm{~d} y,
\end{gathered}
$$

where

$$
H_{p e r}^{1}(Q)=\left\{u \in H_{l o c}^{1}\left(\mathbf{R}^{3}\right), u \text { periodic in } x_{i}, i=1,2,3, \text { of period } 1\right\} .
$$

The main results we obtain in [11] may be stated as follows (we need technical assumptions that are irrelevant in this introduction and that we therefore do not make precise here): up to an additive constant $M / 2$ that only depends on $G$ through

$$
M=\lim _{x \rightarrow 0} G(x)-\frac{1}{|x|},
$$


and which is just a matter of normalization, we have convergence of the TFW energy per unit volume to the infimum $I_{p e r}^{T F W}$; moreover the density $\rho_{\Lambda}$ minimizing $I_{\Lambda}^{T F W}$ converges (uniformly locally, at least) to the unique periodic density $\rho_{\text {per }}$ minimizing $I_{p e r}^{T F W}$.

In view of these results, the reader may understand the main two motivations of our whole work. Our purpose is twofold: first, we want to check that the molecular model under consideration does have the good behaviour in the limit of large volumes; second, we wish to set a limit problem that is well-posed mathematically and that can be justified in the most possible rigorous way (in particular with a view to give a sound ground to the numerical simulations of the condensed phase). As far as this second aim is concerned, it is clear (at least we hope it is) from the above formulae that one keypoint for the definition of the periodic problem is the definition of laws of interaction between particles, i.e. of the interaction potential(s). In the TFW setting, the second aim was less prominent since the potential $G$ is the same as the one appearing in the TF setting and the periodic minimization problem is rather easy to guess in view of the one arising for the TF theory. Likewise, it is easy to check that this periodic minimization problem is mathematically well-posed. In other words, taking benefit from the work by Lieb and Simon who had already defined the TF periodic problem, the idea to introduce the periodic problem (6)-(7) was straightforward. In [11], our "only" contribution was therefore to prove that the TFW model does converge in the thermodynamic limit to (6)(7). The purpose of the present work is the study of the thermodynamic limit problem in the Hartree setting. We shall see below that the guess on the periodic problem is not so obvious in the Hartree model. Consequently, the mere definition of the limit problem turns out to be a substantial piece of the work (writing a periodic problem that has some rigorous mathematical sense is not straightforward). This paper is aimed at describing it. It will certainly be rather clear to the reader that the questions we tackle here in trying to define as rigorously as possible periodic problems in the Hartree framework are indeed close to questions of interest in Solid State Physics, both for theoretical and numerical purposes. For the sake of brevity, we shall not detail here the relationship between our work and Solid State Physics. We only mention some references here, namely [23,40], and also $[2,4,9,39,42,47,48,53]$, and refer the reader to some future work of our own. Because of the complexity of the Hartree setting, we shall not be able to do in this setting everything we did in the TFW setting, namely proving the convergence of the energy per unit volume in the thermodynamic limit. We shall indeed prove the convergence of the energy per unit volume in the thermodynamic limit for a simplified Hartree model (namely the restricted Hartree model, treated in Section 3). Furthermore, we shall prove the convergence of the energy per unit volume for one very peculiar form of the true Hartree model (see Section 4), but our efforts to prove it for the generic form of the Hartree model have failed so far. From the single example we have in hand, and from more general considerations, we shall however deduce a general form for a periodic Hartree problem that is likely to be the thermodynamic limit of the Hartree model. We shall prove, still in Section 4, that this periodic model defines a mathematically wellposed minimization problem.

Let us finally mention that the Hartree-Fock setting is discussed by the authors in [13].

But before all, let us devote Section 2 to the definition of the general setting we shall work in, and to the detailed presentation of the results we shall establish. 


\section{General setting of the models and main results}

Let us begin this section by defining the molecular models we shall deal with in this article. There are two of them, namely on the one hand the Hartree model and, on the other hand, its simplified form, the restricted Hartree model. For the sake of brevity, we shall often abbreviate these models in the $\mathrm{H}$ and the RH models, respectively.

We recall from the introduction that, for each $\Lambda$, finite subset of $\mathbf{Z}^{3} \subset \mathbf{R}^{3}$, we consider the molecular system consisting of the $|\Lambda|$ nuclei located at the points of $\Lambda$, and $|\Lambda|$ electrons. We shall henceforth denote by

$$
V_{\Lambda}(x)=\sum_{k \in \Lambda} \frac{1}{|x-k|}
$$

the attraction potential created by the nuclei on the electrons, and by

$$
\frac{1}{2} U_{\Lambda}=\frac{1}{2} \sum_{m, n \in \Lambda, m \neq n} \frac{1}{|m-n|}
$$

the self-repulsion of the nuclei.

As in [11], we shall also consider the case when the nuclei are not point nuclei but are smeared nuclei. In that case, each Dirac mass located at a point $k$ of $\Lambda$ is replaced by a compactly supported smooth non-negative function of total mass one, typically denoted by $m(\cdot-k)$, and "centered" at that point of $\Lambda$. The regularity of the function $m$ does not play a great role in the sequel, and therefore we shall assume without loss of generality that $m$ is $C^{\infty}$. The potential (9) and the repulsion (10) are then respectively replaced by

$$
\begin{gathered}
V_{\Lambda}^{m}(x)=\sum_{k \in \Lambda} m \star \frac{1}{|x-k|}, \\
\frac{1}{2} U_{\Lambda}^{m}=\frac{1}{2} D\left(\sum_{k \in \Lambda} m(\cdot+k), \sum_{k \in \Lambda} m(\cdot+k)\right)-\frac{1}{2}|\Lambda| D(m, m) .
\end{gathered}
$$

In the above equation, we have as usual denoted by $D(\cdot, \cdot)$ the double integral defined as follows

$$
D(f, f)=\iint_{\mathbf{R}^{3} \mathbf{R}^{3}} \frac{f(x) f(y)}{|x-y|} \mathrm{d} x \mathrm{~d} y .
$$

It will be convenient to introduce in this setting the function

$$
m_{\Lambda}=\sum_{k \in \Lambda} m(\cdot-k)
$$

In this setting of smeared nuclei, we shall also make use of the effective potential $\Phi_{\Lambda}$ defined for each electronic density $\rho_{\Lambda}$ as follows

$$
\Phi_{\Lambda}=\left(m_{\Lambda}-\rho_{\Lambda}\right) \star \frac{1}{|x|} .
$$


It is now time to recall the properties of the sequence of sets $\Lambda$ that we shall consider. For the sake of completeness, we recall here the following definition taken from [11].

DEFINITION 1. - We shall say that a sequence $\left(\Lambda_{i}\right)_{i} \geqslant 1$ of finite subsets of $\mathbf{Z}^{3}$ goes to infinity if the following two conditions hold:

(a) For any finite subset $A \subset \mathbf{Z}^{3}$, there exists $i \in \mathbf{N}$ such that

$$
\forall j \geqslant i, \quad A \subset \Lambda_{j} .
$$

(b) If $\Lambda^{h}$ is the set of points in $\mathbf{R}^{3}$ whose distance to $\partial \Gamma(\Lambda)$ is less than $h$, then

$$
\lim _{i \rightarrow \infty} \frac{\left|\Lambda_{i}^{h}\right|}{\left|\Lambda_{i}\right|}=0, \quad \forall h>0 .
$$

Condition (b) will be hereafter referred to as the Van Hove condition.

Briefly speaking, a sequence satisfying the Van Hove condition is a sequence for which the 'boundary' is negligible in front of the 'interior'. A sequence of large cubes typically satisfies the conditions of Definition 1 . We shall only consider henceforth Van Hove sequences going to infinity in the sense of the above definition. Following the notation of [32,11], we shall write henceforth $\lim _{\Lambda \rightarrow \infty} f(\Lambda)$ instead of $\lim _{i \rightarrow \infty} f\left(\Lambda_{i}\right)$.

We now need to define the following useful functional transformation, that we have already used in [11], and which will be again very efficient in the present work.

DEFINITION 2. - For a given sequence $\Lambda$ and a sequence $\rho_{\Lambda}$ of densities, we call the $\sim$ transform of $\rho_{\Lambda}$ and denote by $\tilde{\rho}_{\Lambda}$ the following sequence of functions

$$
\tilde{\rho}_{\Lambda}=\frac{1}{|\Lambda|} \sum_{k \in \Lambda} \rho_{\Lambda}(\cdot+k) .
$$

We finally introduce

$$
f(x)=\frac{1}{|x|}-\int_{Q} \frac{\mathrm{d} y}{|x-y|},
$$

next

$$
f_{\Lambda}(x)=\sum_{k \in \Lambda}\left(\frac{1}{|x-k|}-\int_{Q} \frac{\mathrm{d} y}{|x-k-y|}\right) .
$$

It is convenient to rewrite $f_{\Lambda}$ as

$$
f_{\Lambda}=V_{\Lambda}-\chi_{\Gamma(\Lambda)} \star \frac{1}{|x|},
$$

where, more generally, we shall denote by $\chi_{\Omega}$ the characteristic function of the domain $\Omega$. Besides, it is proved in [32], and recalled in [11], that, when $Q$ is a cube,

$$
|f(x)| \leqslant \frac{C}{|x|^{4}}
$$


almost everywhere on $\mathbf{R}^{3}$, for some positive constant $C$, and that $f_{\Lambda}$ converges to the periodic potential $G+d$, for some real constant $d$ independent of $\Lambda$, uniformly on compact subsets of $\mathbf{R}^{3} \backslash \mathbf{Z}^{3}$. Moreover, for any compact subset $K$ of $\mathbf{R}^{3}$, $f_{\Lambda}-\sum_{k \in \Lambda \cap K} \frac{1}{|x-k|}$ converges uniformly on $K$ to $G+d-\sum_{k \in \mathbf{Z}^{3} \cap K} \frac{1}{|x-k|}$ (see [32]).

We shall make use in the sequel of the following notation. If $H$ is a functional space, we denote by $H_{\text {unif }}\left(\mathbf{R}^{3}\right)$ the space

$$
H_{u n i f}\left(\mathbf{R}^{3}\right)=\left\{\psi \in \mathcal{D}^{\prime}\left(\mathbf{R}^{3}\right) / \psi \in H(x+Q) \forall x \in \mathbf{R}^{3}, \sup _{x \in \mathbf{R}^{3}}\|\psi\|_{H(x+Q)}<\infty\right\} .
$$

In addition, we shall also simply write $f \star_{Q} g$ instead of $f \star\left(\chi_{Q} g\right)$.

We are now in position to introduce the molecular models we shall deal with. The Hartree model is defined as follows.

$$
\begin{gathered}
I_{\Lambda}^{H}=\inf \left\{E_{\Lambda}^{H}\left(\varphi_{1} ; \ldots ; \varphi_{|\Lambda|}\right)+\frac{1}{2} U_{\Lambda} ; \varphi_{i} \in H^{1}\left(\mathbf{R}^{3}\right), \int_{\mathbf{R}^{3}} \varphi_{i}^{2}=1,1 \leqslant i \leqslant|\Lambda|\right\}, \\
E_{\Lambda}^{H}\left(\varphi_{1} ; \ldots ; \varphi_{|\Lambda|}\right)=\sum_{i=1}^{|\Lambda|}\left(\int_{\mathbf{R}^{3}}\left|\nabla \varphi_{i}\right|^{2}-\frac{1}{2} D\left(\left|\varphi_{i}\right|^{2},\left|\varphi_{i}\right|^{2}\right)\right)-\int_{\mathbf{R}^{3}} V_{\Lambda} \rho+\frac{1}{2} D(\rho, \rho),
\end{gathered}
$$

with

$$
\rho=\sum_{i=1}^{|\Lambda|}\left|\varphi_{i}\right|^{2}
$$

The Hartree model was historically introduced by Hartree in [19]. It is a well-known fact that, for any subset $\Lambda$ of $\mathbf{R}^{3}$, this minimization problem is attained by at least one vector $\left(\varphi_{1} ; \ldots ; \varphi_{|\Lambda|}\right)$, with $\varphi_{i}>0$ for every $1 \leqslant i \leqslant|\Lambda|$ (see the works by Lieb and Simon in [33] and by Lions in [35]).

In the smeared nuclei case, the energy functional of the Hartree model reads as follows

$$
E_{\Lambda}^{m, H}\left(\varphi_{1} ; \ldots ; \varphi_{|\Lambda|}\right)=\sum_{i=1}^{|\Lambda|}\left(\int_{\mathbf{R}^{3}}\left|\nabla \varphi_{i}\right|^{2}-\frac{1}{2} D\left(\left|\varphi_{i}\right|^{2},\left|\varphi_{i}\right|^{2}\right)\right)-\int_{\mathbf{R}^{3}} V_{\Lambda}^{m} \rho+\frac{1}{2} D(\rho, \rho),
$$

and the minimization problem can therefore be written in the following more concise form

$$
\begin{gathered}
I_{\Lambda}^{m, H}=\inf \left\{\sum_{i=1}^{|\Lambda|}\left(\int_{\mathbf{R}^{3}}\left|\nabla \varphi_{i}\right|^{2}-\frac{1}{2} D\left(\left|\varphi_{i}\right|^{2},\left|\varphi_{i}\right|^{2}\right)\right)+\frac{1}{2} D\left(\rho-m_{\Lambda}, \rho-m_{\Lambda}\right)\right. \\
\left.-\frac{1}{2}|\Lambda| D(m, m) ; \varphi_{i} \in H^{1}\left(\mathbf{R}^{3}\right), \int_{\mathbf{R}^{3}} \varphi_{i}^{2}=1,1 \leqslant i \leqslant|\Lambda|\right\},
\end{gathered}
$$

where we recall that $m_{\Lambda}$ is given by (14).

As announced above, we also define the restricted Hartree model, obtained from the standard Hartree model by introducing the self-interaction between electron $i$ and itself 
in the energy functional. In the point nuclei case, this model reads

$$
\begin{gathered}
I_{\Lambda}^{R H}=\inf \left\{E_{\Lambda}^{R H}\left(\varphi_{1} ; \ldots ; \varphi_{|\Lambda|}\right)+\frac{1}{2} U_{\Lambda} ; \varphi_{i} \in H^{1}\left(\mathbf{R}^{3}\right), \int_{\mathbf{R}^{3}} \varphi_{i}^{2}=1,1 \leqslant i \leqslant|\Lambda|\right\}, \\
E_{\Lambda}^{R H}\left(\varphi_{1} ; \ldots ; \varphi_{|\Lambda|}\right)=\int_{\mathbf{R}^{3}} \sum_{i=1}^{|\Lambda|}\left|\nabla \varphi_{i}\right|^{2}-\int_{\mathbf{R}^{3}} V_{\Lambda} \rho+\frac{1}{2} D(\rho, \rho),
\end{gathered}
$$

with $\rho$ being defined as in (21). It is obvious that, for all $\Lambda$,

$$
E_{\Lambda}^{R H} \geqslant E_{\Lambda}^{H},
$$

and thus

$$
I_{\Lambda}^{R H} \geqslant I_{\Lambda}^{H} .
$$

In the smeared nuclei case, the energy functional of the restricted Hartree model reads as follows

$$
E_{\Lambda}^{m, R H}\left(\varphi_{1} ; \ldots ; \varphi_{|\Lambda|}\right)=\sum_{i=1}^{|\Lambda|} \int_{\mathbf{R}^{3}}\left|\nabla \varphi_{i}\right|^{2}-\int_{\mathbf{R}^{3}} V_{\Lambda}^{m} \rho+\frac{1}{2} D(\rho, \rho),
$$

and the minimization problem can therefore be written in the following more concise form

$$
\begin{gathered}
I_{\Lambda}^{m, R H}=\inf \left\{\sum_{i=1}^{|\Lambda|} \int_{\mathbf{R}^{3}}\left|\nabla \varphi_{i}\right|^{2}+\frac{1}{2} D\left(\rho-m_{\Lambda}, \rho-m_{\Lambda}\right)-\frac{1}{2}|\Lambda| D(m, m) ;\right. \\
\left.\varphi_{i} \in H^{1}\left(\mathbf{R}^{3}\right), \int_{\mathbf{R}^{3}} \varphi_{i}^{2}=1,1 \leqslant i \leqslant|\Lambda|\right\} .
\end{gathered}
$$

In view of the periodic problem that we have obtained in [11] for the TFW model, it is rather natural to introduce the following minimization problem, that we intend to relate with the Hartree model with $\Lambda$ fixed:

$$
I_{p e r}^{H}=\inf \left\{E_{p e r}^{H}(\varphi) ; \varphi \in H^{1}\left(\mathbf{R}^{3}\right), \int_{\mathbf{R}^{3}}|\varphi|^{2}=1\right\},
$$

where the periodic energy $E^{H}$ is defined as follows

$$
E_{p e r}^{H}(\varphi)=\int_{\mathbf{R}^{3}}|\nabla \varphi|^{2}-\frac{1}{2} D\left(|\varphi|^{2},|\varphi|^{2}\right)-\int_{Q} G \rho+\frac{1}{2} D_{G}(\rho, \rho),
$$

with

$$
\rho(x)=\sum_{k \in \mathbf{Z}^{3}}|\varphi|^{2}(x+k),
$$


and the following notation that we shall adopt henceforth (in the spirit of the notation (13))

$$
D_{G}(f, f)=\iint_{Q} f(x) G(x-y) f(y) \mathrm{d} x \mathrm{~d} y .
$$

We recall that $Q$ denotes here and henceforth the unit cube $\left.]-\frac{1}{2},+\frac{1}{2}\right]^{3}$. On the other hand, for the restricted Hartree problem, we introduce the following minimization problem

$$
I_{p e r}^{R H}=\inf \left\{E_{p e r}^{R H}(\rho) ; \rho \geqslant 0, \sqrt{\rho} \in H_{p e r}^{1}(Q), \int_{Q} \rho=1\right\},
$$

where we denote by $H_{p e r}^{1}(Q)$ the set of all $Q$-periodic functions in $H_{l o c}^{1}\left(\mathbf{R}^{3}\right)$ and where the periodic energy functional $E^{R H}$ is given by

$$
E_{p e r}^{R H}(\rho)=\int_{Q}|\nabla \sqrt{\rho}|^{2}-\int_{Q} G \rho+\frac{1}{2} D_{G}(\rho, \rho) .
$$

It is easy to show that the minimization problem (34)-(35) admits a unique minimum (the same property will hold true in the smeared nuclei setting below). We now define the periodic $\mathrm{H}$ and $\mathrm{RH}$ problems in the smeared nuclei case.

$$
I_{p e r}^{m, H}=\inf \left\{E_{p e r}^{m, H}(\varphi) ; \varphi \in H^{1}\left(\mathbf{R}^{3}\right), \int_{\mathbf{R}^{3}}|\varphi|^{2}=1\right\},
$$

where the periodic energy $E_{p e r}^{m, H}$ is defined as follows

$$
E_{p e r}^{m, H}(\varphi)=\int_{\mathbf{R}^{3}}|\nabla \varphi|^{2}-\frac{1}{2} D\left(|\varphi|^{2},|\varphi|^{2}\right)+\frac{1}{2} D_{G}(\rho-m, \rho-m)-\frac{1}{2} D_{G}(m, m),
$$

with the periodic density $\rho$ being related to $\varphi$ through (32).

On the other hand, for the restricted Hartree problem, we introduce the following minimization problem

$$
\begin{aligned}
I_{p e r}^{m, R H} & =\inf \left\{E_{p e r}^{m, R H}(\rho) ; \rho \geqslant 0, \sqrt{\rho} \in H_{p e r}^{1}(Q), \int_{Q} \rho=1\right\}, \\
E_{p e r}^{m, R H}(\rho) & =\int_{Q}|\nabla \sqrt{\rho}|^{2}+\frac{1}{2} D_{G}(\rho-m, \rho-m)-\frac{1}{2} D_{G}(m, m) .
\end{aligned}
$$

The main purpose of Section 3 will be to prove the following result on the thermodynamic limit of the $\mathrm{RH}$ problem. 
THEOREM 2.1 (Thermodynamic limit for the RH energy). - In the point nuclei case, we have

$$
\lim _{\Lambda \rightarrow \infty} \frac{I_{\Lambda}^{R H}}{|\Lambda|}=I_{p e r}^{R H}+\frac{M}{2},
$$

where the constant $M$ is defined by (8). Likewise, in the smeared nuclei case, we have

$$
\lim _{\Lambda \rightarrow \infty} \frac{I_{\Lambda}^{m, R H}}{|\Lambda|}=I_{p e r}^{m, R H}+\frac{M}{2},
$$

where $M$ is this time defined by

$$
M=\iint_{Q \times Q} m(x) m(y)[G(x-y)-1 /|x-y|] \mathrm{d} x \mathrm{~d} y .
$$

We shall also make there some comments on this result.

As far as the Hartree model is concerned, we shall extensively present our point of view in Section 4, but let us already emphasize here that our main result will be the following one, which states that the minimization problem we have defined above is mathematically well-posed.

THEOREM 2.2 (Well-posedness of the $\mathrm{H}$ periodic problem). - The minimization problem defined by (30)-(31) (respectively by (36)-(37)) admits a minimum. In addition, any minimizing sequence of (30)-(31) (respectively (36)-(37)) is relatively compact in $H^{1}\left(\mathbf{R}^{3}\right)$, up to a translation.

Is is to be mentioned here that in the proof of the above theorem, we shall make use of the concentration-compactness method [34].

As announced in the introduction, we shall also see in Section 4 that, for a very particular choice of smeared nuclei, we are able to prove the convergence of the Hartree energy per unit volume to the periodic energy (30). We refer the reader to Proposition 4.1 below. We also prove in Section 4.4 the following.

Proposition 2.1. - We assume that the Van Hove sequence $\Lambda$ satisfies

$$
\lim _{\Lambda \rightarrow \infty} \frac{\left|\Lambda^{h}\right|}{|\Lambda|} \log \left|\Lambda^{h}\right|=0, \quad \forall h>0,
$$

where $\Lambda^{h}$ is defined in Definition 1. We assume here that the unit cell $Q$ is a cube and that there exists a minimizer $\varphi_{\text {per }} \in H^{1}\left(\mathbf{R}^{3}\right)$ of $I_{\text {per }}^{H}$ which shares the symmetries of the unit cube. Then,

$$
\limsup _{\Lambda \rightarrow \infty} \frac{I_{\Lambda}^{H}}{|\Lambda|} \leqslant I_{\text {per }}^{H}+\frac{M}{2},
$$

where $I_{\text {per }}^{H}$ is defined by (30)-(31).

As announced in the introduction, the sequel of this paper is devoted to the proofs of the above results. We shall also give some complements. 


\section{The restricted Hartree model}

We devote this section to the thermodynamic limit problem of the so-called restricted Hartree model (RH model for short). We shall see that we shall be allowed to extend to this setting most of the methods introduced in [11] in order to prove that the TFW energy has a thermodynamic limit. Of course this study can be seen as a step towards the study of the complete Hartree model ( $\mathrm{H}$ for short) that will be addressed in the following section. We shall see however that despite their relative formal resemblance, the $\mathrm{RH}$ model, on the one hand, and the Hartree model, on the other hand, do behave in a very different fashion, as far as the thermodynamic limit problem is concerned. For the time being, let us concentrate on the RH model.

Let us now recall the definition we have given in Section 2 above of the restricted Hartree model. For the sake of brevity, we shall only consider in this section the case of point nuclei. Actually, the case of smeared nuclei is easier to treat, and we leave it to the reader.

For every finite subset $\Lambda$ of $\mathbf{Z}^{3}$, the RH model is defined as follows:

with

$$
\begin{aligned}
I_{\Lambda}^{R H}=\inf \{ & E_{\Lambda}^{R H}\left(\varphi_{1} ; \ldots ; \varphi_{|\Lambda|}\right)+\frac{1}{2} U_{\Lambda} ; \\
& \left.\forall 1 \leqslant i \leqslant|\Lambda|, \varphi_{i} \in H^{1}\left(\mathbf{R}^{3}\right), \int_{\mathbf{R}^{3}} \varphi_{i}^{2}=1\right\},
\end{aligned}
$$

$$
E_{\Lambda}^{R H}\left(\varphi_{1} ; \ldots ; \varphi_{|\Lambda|}\right)=\int_{\mathbf{R}^{3}} \sum_{i=1}^{|\Lambda|}\left|\nabla \varphi_{i}\right|^{2}-\int_{\mathbf{R}^{3}} V_{\Lambda} \rho+\frac{1}{2} \iint_{\mathbf{R}^{3} \times \mathbf{R}^{3}} \frac{\rho(x) \rho(y)}{|x-y|} \mathrm{d} x \mathrm{~d} y,
$$

$\rho=\sum_{i=1}^{|\Lambda|}\left|\varphi_{i}\right|^{2}$, and where we recall that $V_{\Lambda}(x)=\sum_{y \in \Lambda} \frac{1}{|x-y|}$. If we compare with the complete Hartree model given in (19)-(20), we may note that only the interaction between the electrons has been modified and has been replaced by a mean-field potential which is the same for each of the $|\Lambda|$ electrons. In other words, the self-interaction of each electron has been reincorporated into the energy functional.

We show now that, due to this modification, this infimum is the same as

$$
\inf \left\{E_{\Lambda}^{R H}(\rho)+\frac{1}{2} U_{\Lambda} ; \rho \geqslant 0, \sqrt{\rho} \in H^{1}\left(\mathbf{R}^{3}\right), \int_{\mathbf{R}^{3}} \rho=|\Lambda|\right\},
$$

with

$$
E_{\Lambda}^{R H}(\rho)=\int_{\mathbf{R}^{3}}|\nabla \sqrt{\rho}|^{2}-\int_{\mathbf{R}^{3}} V_{\Lambda} \rho+\frac{1}{2} \iint_{\mathbf{R}^{3} \times \mathbf{R}^{3}} \frac{\rho(x) \rho(y)}{|x-y|} \mathrm{d} x \mathrm{~d} y .
$$

Indeed, we first recall from [35] that, on the one hand, the infimum defined by (44)(45) is achieved by a unique positive function $\rho^{R H}$ (the uniqueness coming from the strict convexity of the functional $\rho \mapsto E_{\Lambda}^{R H}(\rho)$ defined by (45)). On the other hand, the 
infimum in (42) is attained by $|\Lambda|$ positive functions $\varphi_{i}$, for $1 \leqslant i \leqslant|\Lambda|$. In addition, for every $1 \leqslant i \leqslant|\Lambda|, \varphi_{i}$ satisfies

$$
-\Delta \varphi_{i}-V_{\Lambda} \varphi_{i}+\left(\rho \star \frac{1}{|x|}\right) \varphi_{i}+\theta_{i} \varphi_{i}=0 \quad \text { on } \mathbf{R}^{3},
$$

for some $\theta_{i}>0$. This latter claim comes from the fact that, since $\int \rho=|\Lambda|$, the positive part of the spherical average of the potential $-V_{\Lambda}+\left(\rho \star \frac{1}{|x|}\right)$, which is identically 0 , lies in $L^{3 / 2}\left(\mathbf{R}^{3}\right)$. Then, we may apply a result of Lieb and Simon in [32]. Therefore, since $\varphi_{i}>0$ and since $-V_{\Lambda}+\left(\rho \star \frac{1}{|x|}\right)$ also belongs to $L_{\text {unif }}^{p}\left(\mathbf{R}^{3}\right)$, for some $p>\frac{3}{2}$, $\varphi_{i}$ is the (unique) positive normalized eigenfunction associated to the first eigenvalue of the operator $-\Delta-V_{\Lambda}+\left(\rho \star \frac{1}{|x|}\right)$ on $\mathbf{R}^{3}$, and the corresponding eigenspace is of dimension 1 (see, for example, [46]). We thus conclude that $\theta_{1}=\cdots=\theta_{|\Lambda|}$ and $\varphi_{1}=\cdots=\varphi_{|\Lambda|}\left(=\frac{1}{\sqrt{|\Lambda|}} \sqrt{\rho}\right)$. Then, returning to (46), we deduce that $\varphi=\sqrt{\rho}$ is a critical point for $E_{\Lambda}^{R H}$. Since the functional $\rho \mapsto E_{\Lambda}^{R H}(\rho)$ is strictly convex and since $\rho$ satisfies the right charge constraint, we conclude that $\rho$ is the unique minimizer of $E_{\Lambda}^{R H}$, that is $\rho^{R H}$. Our claim follows.

From now on, with a view to proving the existence of the thermodynamic limit for the energy per unit volume for the $R H$ model, we shall essentially use the expression (45) for the energy and identify $I_{\Lambda}^{R H}$ with (44). It is therefore to be emphasized that we deal with a sequence of minimization problems which are of density functional type: only the electronic density $\rho$ appears in the minimization and not the electronic wavefunctions initially involved in (43). Consequently, we shall be able to use most of the machinery developed in [11] to treat the TFW model. As far as the thermodynamic limit for the energy per unit volume is concerned, this machinery (in particular the trick that consists of approximating the Coulomb problem by a problem where the interaction is of Yukawa type) will be effective and really allows us to determine the behaviour of $E_{\Lambda}^{R H} /|\Lambda|$ (see Theorem 3.1 below). Unfortunately, we have not been able to use it in order to determine the behaviour of the density $\rho_{\Lambda}$, apart from some very basic results that will be mentioned below.

We shall relate the thermodynamic limit of the restricted Hartree model with the periodic minimization problem defined by

$$
I_{p e r}^{R H}=\inf \left\{E_{p e r}^{R H}(\rho) ; \rho \geqslant 0, \sqrt{\rho} \in H_{p e r}^{1}(Q), \int_{Q} \rho=1\right\},
$$

where

$$
E_{p e r}^{R H}(\rho)=\int_{Q}|\nabla \sqrt{\rho}|^{2}-\int_{Q} G \rho+\frac{1}{2} \iint_{Q \times Q} \rho(x) \rho(y) G(x-y) \mathrm{d} x \mathrm{~d} y .
$$

Before turning to the thermodynamic limit problem per se, let us first give some results on the existence and the uniqueness of the minimizer of $I_{p e r}^{R H}$.

LEMMA 3.1 (Properties of $I_{p e r}^{R H}$ ). - Let $I_{p e r}^{R H}$ be defined by (47) and (48). Then, $I_{p e r}^{R H}$ is achieved by a unique positive function $\rho_{p e r}, u_{p e r}=\sqrt{\rho_{p e r}} \in H_{p e r}^{1}(Q) \cap L^{\infty}\left(\mathbf{R}^{3}\right)$, and 
satisfies

$$
-\Delta u_{p e r}-G u_{p e r}+\left(\int_{Q} G(x-y) \rho_{p e r}(y) \mathrm{d} y\right) u_{p e r}+\theta_{p e r} u_{p e r}=0, \quad \text { on } \mathbf{R}^{3},
$$

for some real number $\theta_{\text {per }}$.

Proof of Lemma 3.1. - The existence and the uniqueness of a minimizer of $I_{p e r}^{R H}$ follows from the following observations. Since $G$ is periodic and since the function $G-\frac{1}{|x|}$ is continuous and bounded on $Q$, it is easy to check that $I_{p e r}^{R H}$ is finite. Indeed, on the one hand, it is easily seen, by using for example the Fourier series expansion of the periodic potential $G$ (see [32]), that the quadratic form $f \mapsto D_{G}(f, f)$ is non-negative. On the other hand, since $G$ is in $L_{u n i f}^{3 / 2}\left(\mathbf{R}^{3}\right)$, for every $\varepsilon>0$, there is a positive constant $k(\varepsilon)$ such that we may decompose $G$ into $G=G_{1}+G_{2}$ with $\left\|G_{1}\right\|_{L^{\infty}(Q)} \leqslant k(\varepsilon)$ and $\left\|G_{2}\right\|_{L^{3 / 2}(Q)} \leqslant \varepsilon$. Now let $\rho \geqslant 0$ be such that $u \equiv \sqrt{\rho} \in H_{p e r}^{1}(Q)$. We first notice that $0<\int_{Q} u \leqslant 1$ because $\int_{Q} u^{2}=1$, and from Schwarz's inequality. Therefore, we have, using first Hölder's, and then Sobolev-Poincaré's inequalities,

$$
\begin{aligned}
E_{p e r}^{H}(\rho) & \geqslant \int_{Q}|\nabla u|^{2}-\int_{Q} G(x) u^{2}(x) \mathrm{d} x \\
& \geqslant \int_{Q}|\nabla u|^{2}-k(\varepsilon) \int_{Q} u^{2}-\varepsilon\|u\|_{L^{6}(Q)}^{2} \\
& \geqslant(1-2 \varepsilon) \int_{Q}|\nabla u|^{2}-k(\varepsilon)-C \varepsilon
\end{aligned}
$$

for some positive constant $C$, that is independent of $\varepsilon$ and $u$. We conclude by choosing $\varepsilon$ small enough.

By the way, the same argument shows that every minimizing sequence $\rho_{n}$ of $I_{p e r}^{H}$ is such that $u_{n}=\sqrt{\rho_{n}}$ is bounded in $H_{p e r}^{1}(Q)$. Then, extracting a subsequence if necessary, we may assume that $u_{n}$ converges weakly in $H_{p e r}^{1}(Q)$, strongly in $L_{\text {unif }}^{p}\left(\mathbf{R}^{3}\right)$ for all $1 \leqslant p<6$ (from Rellich's Theorem) and almost everywhere on $\mathbf{R}^{3}$. The limit is then a minimizer of $I_{p e r}^{H}$. The uniqueness of the minimizer follows from the strict convexity of the functional.

In addition, since $G$ is in $L_{\text {unif }}^{q}\left(\mathbf{R}^{3}\right)$ for all $1 \leqslant q<3$, it is clear from (49) that $-\Delta u_{p e r}$ is in $L_{\text {unif }}^{p}$ for every $1 \leqslant p<2$. Thus, $u \in W_{\text {unif }}^{2, p}$. In particular, from Sobolev's embeddings, $u \in L^{\infty}\left(\mathbf{R}^{3}\right)$. In fact, by a standard bootstrap argument, $u$ is in $W_{u n i f}^{2, p} \cap C^{0, \alpha}$, for every $1 \leqslant p<3$ and $0<\alpha<1$.

Let us turn now to the thermodynamic limit problem we are interested in and prove first the following:

LEMmA 3.2. - For every Van Hove sequence $(\Lambda)$, we have

$$
\limsup _{\Lambda \rightarrow \infty} \frac{I_{\Lambda}^{R H}}{|\Lambda|} \leqslant I_{p e r}^{R H}+\frac{M}{2} .
$$


Proof of Lemma 3.2. - The proof is immediate once we have noticed that, for all $\varepsilon>0$,

$$
I_{\Lambda}^{R H} \leqslant I_{\Lambda, \varepsilon}^{T F W},
$$

where the notation $I_{\Lambda, \varepsilon}^{T F W}$ stands for the usual TFW problem we have studied in [11], with $\varepsilon$ as a coefficient in front of the Thomas-Fermi term $\int_{\mathbf{R}^{3}} \rho^{5 / 3}$ in the definition of the TFW functional; namely

$$
\begin{gathered}
E_{\Lambda, \varepsilon}^{T F W}(\rho)=\int_{\mathbf{R}^{3}}|\nabla \sqrt{\rho}|^{2}+\varepsilon \int_{\mathbf{R}^{3}} \rho^{5 / 3}-\int_{\mathbf{R}^{3}}\left(\sum_{k \in \Lambda} \frac{1}{|x-k|}\right) \rho(x) \mathrm{d} x \\
+\frac{1}{2} \int_{\mathbf{R}^{3} \times \mathbf{R}^{3}} \frac{\rho(x) \rho(y)}{|x-y|} \mathrm{d} x \mathrm{~d} y, \\
I_{\Lambda, \varepsilon}^{T F W}=\inf \left\{E_{\Lambda, \varepsilon}^{T F W}(\rho)+\frac{1}{2} \sum_{y \neq z \in \Lambda} \frac{1}{|y-z|} ; \rho \geqslant 0, \sqrt{\rho} \in H^{1}\left(\mathbf{R}^{3}\right), \int_{\mathbf{R}^{3}} \rho=|\Lambda|\right\} .
\end{gathered}
$$

Next, in view of the results of [11], we obtain from (51), and for every $\varepsilon>0$,

$$
\limsup _{\Lambda \rightarrow \infty} \frac{I_{\Lambda}^{H}}{|\Lambda|} \leqslant \lim _{\Lambda \rightarrow \infty} \frac{I_{\Lambda, \varepsilon}^{T F W}}{|\Lambda|}=I_{p e r, \varepsilon}^{T F W}+\frac{M}{2}
$$

where, obviously, $I_{p e r, \varepsilon}^{T F W}$ is the periodic TFW model with a multiplicative parameter $\varepsilon$ in front of the term $\int_{Q} \rho^{5 / 3}$ in the definition of the TFW periodic functional; namely

$$
\begin{gathered}
I_{p e r, \varepsilon}^{T F W}=\inf \left\{E_{p e r, \varepsilon}^{T F W}(\rho) ; \rho \geqslant 0, \sqrt{\rho} \in H_{p e r}^{1}(Q), \int_{Q} \rho=1\right\}, \\
E_{p e r, \varepsilon}^{T F W}(\rho)=\int_{Q}|\nabla \sqrt{\rho}|^{2}+\varepsilon \int_{Q} \rho^{5 / 3}-\int_{Q} \rho(x) G(x) \mathrm{d} x \\
+\frac{1}{2} \iint_{Q \times Q} \rho(x) \rho(y) G(x-y) \mathrm{d} x \mathrm{~d} y .
\end{gathered}
$$

Assertion (50) follows now by letting $\varepsilon$ go to 0 in (54), and by comparing with the definition (47) of $I_{p e r}^{R H}$.

We next prove the existence of a bound from below for the energy per unit volume in the RH case.

LEMmA 3.3. - For every Van Hove sequence $(\Lambda)$, we have

$$
\liminf _{\Lambda \rightarrow \infty} \frac{I_{\Lambda}^{R H}}{|\Lambda|} \geqslant I_{p e r}^{R H}+\frac{M}{2} .
$$


Proof of Lemma 3.3. - Our strategy of proof will consist of comparing from below $I_{\Lambda}^{R H}$ with the corresponding minimization problem where the Coulomb potential has been replaced by a Yukawa potential $\frac{\exp (-a|x|)}{|x|}, a>0$, and then letting $a$ go to 0 . Let us recall that the same strategy has already been used in [11] in the TFW setting. We shall therefore only sketch the main lines of the proof and refer the reader to [11] for the details.

We thus define, for every $a>0$,

$$
I_{\Lambda}^{a}=\inf \left\{E_{\Lambda}^{a}(\rho)+\frac{1}{2} U_{\Lambda}^{a} ; \rho \geqslant 0, \sqrt{\rho} \in H^{1}\left(\mathbf{R}^{3}\right), \int_{\mathbf{R}^{3}} \rho=|\Lambda|\right\},
$$

with

$$
\begin{gathered}
E_{\Lambda}^{a}(\rho)=\int_{\mathbf{R}^{3}}|\nabla \sqrt{\rho}|^{2}-\int_{\mathbf{R}^{3}} V_{\Lambda}^{a} \rho+\frac{1}{2} \iint_{\mathbf{R}^{3} \times \mathbf{R}^{3}} \rho(x) \rho(y) V^{a}(x-y) \mathrm{d} x \mathrm{~d} y, \\
V^{a}(x)=\frac{\exp (-a|x|)}{|x|}, \quad V_{\Lambda}^{a}(x)=\sum_{y \in \Lambda} V^{a}(x-y), \quad \text { and } \quad U_{\Lambda}^{a}=\sum_{\substack{y, z \in \Lambda \\
y \neq z}} V^{a}(y-z) .
\end{gathered}
$$

It is clear that we may choose $a$ small enough such that $I_{\Lambda}^{a}$ is achieved for all finite subset $\Lambda$ of $\mathbf{Z}^{3}$. In addition, by using the methods of Chapter 2 of [11] for the upper limit and the ones of Chapter 3 of [11] for the lower limit, it is easy to check that

$$
\lim _{\Lambda \rightarrow \infty} \frac{I_{\Lambda}^{a}}{|\Lambda|}=I_{p e r}^{a}\left(\mu_{a}\right),
$$

for any Van Hove sequence $(\Lambda)$, where $\mu_{a}$ and $I_{p e r}^{a}\left(\mu_{a}\right)$ are defined just below. We set

$$
I_{p e r}^{a}\left(\mu_{a}\right)=\inf \left\{E_{p e r}^{a}(\rho)+\frac{1}{2} U_{\infty}^{a} ; \rho \geqslant 0, \sqrt{\rho} \in H_{p e r}^{1}(Q), \int_{Q} \rho=\mu_{a}\right\},
$$

with

$$
\begin{gathered}
E_{p e r}^{a}(\rho)=\int_{Q}|\nabla \sqrt{\rho}|^{2}-\int_{Q} V_{\infty}^{a}(x) \rho(x) \mathrm{d} x+\frac{1}{2} \iint_{Q \times Q} \rho(x) \rho(y) V_{\infty}^{a}(x-y) \mathrm{d} x \mathrm{~d} y, \\
V_{\infty}^{a}(x)=\sum_{y \in \mathbf{Z}^{3}} V^{a}(x-y), \quad \text { and } \quad U_{\infty}^{a}=\sum_{\substack{y, z \in \mathbf{Z}^{3} \\
y \neq z}} V^{a}(y-z) .
\end{gathered}
$$

Finally the mass $\mu_{a}$ is defined as follows. We denote by $\rho_{p e r}^{a}$ the unique minimizer of $E_{p e r}^{a}$ on the set $\left\{\rho \geqslant 0, \sqrt{\rho} \in H_{p e r}^{1}(Q)\right\}$. Then, we define $\mu_{a}=\min \left(1, \int_{Q} \rho_{p e r}^{a}\right)$. (All these definitions are justified in [11].) Arguing as in Chapter 2 of [11], we may prove that

$$
\lim _{a \rightarrow 0^{+}} \mu_{a}=1
$$


and that

$$
\lim _{a \rightarrow 0^{+}} I_{p e r}^{a}\left(\mu_{a}\right)=I_{p e r}^{R H}+\frac{M}{2} .
$$

To conclude, we argue now as in Chapter 3 of [11], to check that

$$
\frac{I_{\Lambda}^{R H}}{|\Lambda|} \geqslant \frac{I_{\Lambda}^{a}}{|\Lambda|}-C a
$$

for some positive constant $C$ that is independent of $\Lambda$. Next, we let $\Lambda$ go to infinity in the above inequality and use (60) to obtain

$$
\liminf _{\Lambda \rightarrow \infty} \frac{I_{\Lambda}^{R H}}{|\Lambda|} \geqslant I_{p e r}^{a}\left(\mu_{a}\right)-C a .
$$

(57) then follows by letting $a$ go to 0 and by using (61).

Remark 3.1. - In the case when the unit cell is a cube, it is possible to prove the above lemma by a different argument which does not use the comparison with a Yukawa potential. Indeed, as in [11], we may use the $\sim$-transform trick and prove directly the lower bound. Of course, this argument relies upon the convexity of the RH functional with respect to the electronic density.

As a consequence of (50), we may prove that

COROLlARY 3.1 (Compactness). - Let $\rho_{\Lambda}$ be the minimizer of $I_{\Lambda}^{R H}$, then

$$
\frac{1}{|\Lambda|} \int_{\Gamma(\Lambda)^{c}} \rho_{\Lambda} \rightarrow 0, \quad \text { as } \Lambda \rightarrow \infty .
$$

The analogous result holds true in the Hartree setting, and a proof is sketched in this setting (see the proof of Lemma 4.3 below).

Remark 3.2. - This property means that, asymptotically, the $|\Lambda|$ electrons remain in $\Gamma(\Lambda)$; that is, in a box of volume $|\Lambda|$. In other words, we could also say that no electrons have escaped to "infinity"; this is the reason why this property is referred to as "compactness" in [11].

Finally, collecting Lemma 3.2 and Lemma 3.3, we have proved the following

THEOREM 3.1 (Thermodynamic limit for the energy in the RH model). - For every Van Hove sequence $(\Lambda)$,

$$
\lim _{\Lambda \rightarrow \infty} \frac{I_{\Lambda}^{R H}}{|\Lambda|}=I_{\text {per }}^{R H}+\frac{M}{2} .
$$

Let us make some comments. Having proved the existence of the thermodynamic limit for the energy per unit volume for the RH model, we may prove as in Chapter 5 of [11], some preliminary convergence results concerning the convergence of the densities. In particular, we may show that the $\sim$-transform of $\rho_{\Lambda}, \tilde{\rho}_{\Lambda}$, converges to the minimizer $\rho_{\text {per }}$ 
of $I_{\text {per }}^{H}$, or, even, that $\rho_{\Lambda}\left(\cdot+y_{\Lambda}\right)$ converges to $\rho_{\text {per }}$ in $H^{1}(Q)$ for some $y_{\Lambda} \in \Lambda$. However, we do not know how to improve these (weak) notions of convergence of the densities. Indeed, in the framework of the RH model, we are not able to establish uniform $L^{\infty}$ bounds (for example) for the densities as in the TFW case, since the bounds obtained in that setting are based upon the specific nature of the power nonlinearity which arises in the Euler-Lagrange equations (see [11] for the details). Another point which is related to the previous one is that we do not know whether there exists a unique solution $(u, \Phi)$ to the system

$$
\left\{\begin{array}{l}
-\Delta u-\Phi u=0 \quad \text { on } \mathbf{R}^{3}, \\
u \geqslant 0, \quad u \neq \equiv 0, \\
-\Delta \Phi=4 \pi\left[\sum_{k \in \mathbf{Z}^{3}} \delta_{k}-u^{2}\right],
\end{array}\right.
$$

without prescribing boundary conditions on $u$ or $\Phi$ (like periodicity or conditions at infinity, for example). On the contrary, we have proved in [11] that the analogue of the above system in the TFW setting, which can be written as

$$
\left\{\begin{array}{l}
-\Delta u+u^{7 / 3}-\Phi u=0, \\
u \geqslant 0, \quad u \neq \equiv 0, \\
-\Delta \Phi=4 \pi\left[\sum_{k \in \mathbf{Z}^{3}} \delta_{k}-u^{2}\right],
\end{array}\right.
$$

admits a unique (thus periodic) solution.

\section{The Hartree model}

Let us first of all recall the Hartree model which will be the subject of this section:

$$
\begin{gathered}
I_{\Lambda}^{H}=\inf \left\{E_{\Lambda}^{H}\left(\varphi_{1} ; \ldots ; \varphi_{|\Lambda|}\right)+\frac{1}{2} U_{\Lambda} ; \varphi_{i} \in H^{1}\left(\mathbf{R}^{3}\right), \int_{\mathbf{R}^{3}} \varphi_{i}^{2}=1,1 \leqslant i \leqslant|\Lambda|\right\}, \\
E_{\Lambda}^{H}\left(\varphi_{1} ; \ldots ; \varphi_{|\Lambda|}\right)=\sum_{i=1}^{|\Lambda|}\left(\int_{\mathbf{R}^{3}}\left|\nabla \varphi_{i}\right|^{2}-\frac{1}{2} D\left(\left|\varphi_{i}\right|^{2},\left|\varphi_{i}\right|^{2}\right)\right)-\int_{\mathbf{R}^{3}} V_{\Lambda} \rho+\frac{1}{2} D(\rho, \rho),
\end{gathered}
$$

where as usual we denote $\rho=\sum_{i=1}^{|\Lambda|}\left|\varphi_{i}\right|^{2}$.

In view of the energy functional (63) which clearly is a sum of a functional involving the $\varphi_{i}$ 's explicitly and a functional depending only on the density $\rho$ and not on the $\varphi_{i}$ 's themselves, it is rather natural to isolate the part depending explicitly on the $\varphi_{i}$ 's, and therefore to introduce the following auxiliary minimization problem

$$
I_{C}=\inf \left\{E_{C}(\varphi) ; \varphi \in H^{1}\left(\mathbf{R}^{3}\right), \int_{\mathbf{R}^{3}}|\varphi|^{2}=1\right\},
$$


with

$$
E_{C}(\varphi)=\int_{\mathbf{R}^{3}}|\nabla \varphi|^{2}-\frac{1}{2} D\left(|\varphi|^{2},|\varphi|^{2}\right),
$$

which is nothing else but the well-known Choquard problem. For the convenience of the reader, we mention here that the fact that $I_{C}>-\infty$ is a straightforward consequence of the following string of inequalities

$$
\begin{aligned}
D\left(\varphi^{2}, \varphi^{2}\right) & \leqslant C\left\|\varphi^{2}\right\|_{L^{6 / 5}\left(\mathbf{R}^{3}\right)}^{2}=C\|\varphi\|_{L^{12 / 5}\left(\mathbf{R}^{3}\right)}^{4} \\
& \leqslant C\|\varphi\|_{L^{2}\left(\mathbf{R}^{3}\right)}^{3}\|\varphi\|_{L^{6}\left(\mathbf{R}^{3}\right)} \leqslant C\|\nabla \varphi\|_{L^{2}\left(\mathbf{R}^{3}\right)},
\end{aligned}
$$

where $C$ denotes various positive constants that are independent of $\varphi$, the last inequality being true since $\int_{\mathbf{R}^{3}} \varphi^{2}=1$. The existence and the uniqueness of the minimizer of (65) are subtler facts proven in Lieb [28].

As a matter of fact, the Choquard problem will play a central role in our analysis of the thermodynamic limit for the Hartree energy. We shall see that below, but let us for the moment concentrate ourselves on the a priori estimates that we may show on the Hartree energy per unit volume.

\subsection{A priori estimates and consequences}

The first estimate is rather straightforward, since it is a simple consequence of Lemma 3.2 and of the fact that the Hartree energy functional is clearly bounded from above by the restricted Hartree energy functional:

LEMmA 4.1. - There exists a constant $C$ such that, for any Van Hove sequence $(\Lambda)$,

$$
\frac{I_{\Lambda}^{H}}{|\Lambda|} \leqslant C
$$

Let us now turn to the existence of lower bound for the energy per unit volume. Of course, we cannot use any more the analogous results for the restricted Hartree model. Nevertheless, we have:

Lemma 4.2. - There exists a constant $C$ such that, for any Van Hove sequence $(\Lambda)$,

$$
\frac{I_{\Lambda}^{H}}{|\Lambda|} \geqslant C
$$

Proof of Lemma 4.2. - Let us first prove this claim in the case of smeared nuclei, and we shall next explain how we proceed with minor modifications in the case of point nuclei.

We recall the expression (22)-(23) for the energy in the case of smeared nuclei

$$
\begin{aligned}
E_{\Lambda}^{m, H}\left(\varphi_{1} ; \ldots ; \varphi_{|\Lambda|}\right)= & \sum_{i=1}^{|\Lambda|}\left[\int_{\mathbf{R}^{3}}\left|\nabla \varphi_{i}\right|^{2}-\frac{1}{2} D\left(\varphi_{i}^{2}, \varphi_{i}^{2}\right)\right] \\
& +\frac{1}{2} D\left(m_{\Lambda}-\rho_{\Lambda}, m_{\Lambda}-\rho_{\Lambda}\right)-\frac{1}{2}|\Lambda| D(m, m) .
\end{aligned}
$$


Since the term $D\left(m_{\Lambda}-\rho_{\Lambda}, m_{\Lambda}-\rho_{\Lambda}\right)$ is non-negative, it follows that

$$
\begin{aligned}
\frac{1}{|\Lambda|} E_{\Lambda}^{m, H}\left(\varphi_{1} ; \ldots ; \varphi_{|\Lambda|}\right) & \geqslant \frac{1}{|\Lambda|} \sum_{i=1}^{|\Lambda|}\left[\int_{\mathbf{R}^{3}}\left|\nabla \varphi_{i}\right|^{2}-\frac{1}{2} D\left(\varphi_{i}^{2}, \varphi_{i}^{2}\right)\right]-\frac{1}{2} D(m, m) \\
& \geqslant I_{C}-\frac{1}{2} D(m, m),
\end{aligned}
$$

using the definition that we have recalled above of the Choquard problem.

In the case of point nuclei, arguing as in Chapter 3 of [11], we rewrite the expression for $I_{\Lambda}^{H}=E_{\Lambda}^{H}\left(\varphi_{1}^{H} ; \ldots ; \varphi_{|\Lambda|}^{H}\right)+\frac{U_{\Lambda}}{2}$ as

$$
\begin{aligned}
I_{\Lambda}^{H}= & \sum_{i=1}^{|\Lambda|}\left[\int_{\mathbf{R}^{3}}\left|\nabla \varphi_{i}\right|^{2}-\frac{1}{2} D\left(\varphi_{i}^{2}, \varphi_{i}^{2}\right)\right]-\int_{\mathbf{R}^{3}} f_{\Lambda} \rho_{\Lambda} \\
& +\frac{1}{2} D\left(\chi_{\Gamma(\Lambda)}-\rho_{\Lambda}, \chi_{\Gamma(\Lambda)}-\rho_{\Lambda}\right)+\frac{1}{2} U_{\Lambda}-\frac{1}{2} D\left(\chi_{\Gamma(\Lambda)}, \chi_{\Gamma(\Lambda)}\right),
\end{aligned}
$$

where $f_{\Lambda}$ is defined through (16). As before, we begin with noticing that the first sum is bounded from below as follows

$$
\sum_{i=1}^{|\Lambda|}\left[\int_{\mathbf{R}^{3}}\left|\nabla \varphi_{i}\right|^{2}-\frac{1}{2} D\left(\varphi_{i}^{2}, \varphi_{i}^{2}\right)\right] \geqslant|\Lambda| I_{C},
$$

and that the term $\frac{1}{2} D\left(\chi_{\Gamma(\Lambda)}-\rho_{\Lambda}, \chi_{\Gamma(\Lambda)}-\rho_{\Lambda}\right)$ is non-negative. Therefore proving the bound from below amounts to proving that

$$
-\int_{\mathbf{R}^{3}} f_{\Lambda} \rho_{\Lambda}+\frac{1}{2} U_{\Lambda}-\frac{1}{2} D\left(\chi_{\Gamma(\Lambda)}, \chi_{\Gamma(\Lambda)}\right) \geqslant-C|\Lambda|,
$$

where, here and below, $C$ denotes a positive constant that is independent of $\Lambda$. On the one hand, we have already proved in [11] that

$$
\left|U_{\Lambda}-D\left(\chi_{\Gamma(\Lambda)}, \chi_{\Gamma(\Lambda)}\right)\right| \leqslant C|\Lambda| .
$$

On the other hand, in view of the upper bound (68) on $I_{\Lambda}^{H}$, it is straightforward to see that

$$
\sum_{i=1}^{|\Lambda|}\left[\int_{\mathbf{R}^{3}}\left|\nabla \varphi_{i}\right|^{2}-\frac{1}{2} D\left(\varphi_{i}^{2}, \varphi_{i}^{2}\right)\right]-\int_{\mathbf{R}^{3}} f_{\Lambda} \rho_{\Lambda} \leqslant C|\Lambda|,
$$

We next show, without difficulty, that for all $\varepsilon>0$, there exists a positive constant $k(\varepsilon)$ independent of $\Lambda$ such that $f_{\Lambda}=f_{\Lambda}^{(1)}+f_{\Lambda}^{(2)}$ with $\left\|f_{\Lambda}^{(1)}\right\|_{L^{\infty}} \leqslant k(\varepsilon)$ and $\left\|f_{\Lambda}^{(2)}\right\|_{L_{\text {unif }}^{3 / 2}} \leqslant \varepsilon$. Then, using Hölder's and Sobolev's inequality, we check that

$$
\left|\int_{\mathbf{R}^{3}} f_{\Lambda} \rho_{\Lambda}\right| \leqslant k(\varepsilon)|\Lambda|+\varepsilon \sum_{i=1}^{|\Lambda|} \int_{\mathbf{R}^{3}}\left|\nabla \varphi_{i}\right|^{2} .
$$


At this stage, we insert, for every $\varepsilon>0$, the bound (75) into (74). It follows that

$$
\frac{1}{|\Lambda|} \sum_{i=1}^{|\Lambda|} \int_{\mathbf{R}^{3}}\left|\nabla \varphi_{i}\right|^{2} \leqslant C
$$

by the same argument as the one used to establish (67), and that

$$
\frac{1}{|\Lambda|} \sum_{i=1}^{|\Lambda|} D\left(\varphi_{i}^{2}, \varphi_{i}^{2}\right) \leqslant C
$$

Therefore, we have

$$
\left|\int_{\mathbf{R}^{3}} f_{\Lambda} \rho_{\Lambda}\right| \leqslant C|\Lambda|
$$

and consequently, returning to (72), the desired bound from below follows.

Thanks to the bounds we have obtained in the course of the proof of the above lemma, we state (and prove) in the following two lemmas, first a "compactness" result which is similar to the one we have obtained in the RH setting (in particular, the comments in Remark 3.2 also apply here), and, next, a further bound on the electronic density.

LEMMA 4.3. - Let $\rho_{\Lambda}$ be the electronic density corresponding to a minimizer $\left(\varphi_{1, \Lambda}^{H} ; \ldots ; \varphi_{|\Lambda|, \Lambda}^{H}\right)$ of $I_{\Lambda}^{H}$. We have

$$
\lim _{\Lambda \rightarrow \infty} \frac{1}{|\Lambda|} \int_{\Gamma(\Lambda)^{c}} \rho_{\Lambda}=0
$$

With the help of the bounds (80) and (76), we may establish the

LEMMA 4.4. - Let $\rho_{\Lambda}$ be the electronic density corresponding to a minimizer $\left(\varphi_{1, \Lambda}^{H} ; \ldots ; \varphi_{|\Lambda|, \Lambda}^{H}\right)$ of $I_{\Lambda}^{H}$. We have

$$
\frac{1}{|\Lambda|} \int_{\mathbf{R}^{3}} \rho_{\Lambda}^{3 / 2} \leqslant C
$$

for some constant $C$ that is independent of $\Lambda$.

Proof of Lemmas 4.3 and 4.4. - Let us argue first in the smeared nuclei case. We recall from [49] and [11] that, for any function $h_{\Lambda}$ in $H^{1}\left(\mathbf{R}^{3}\right)$,

$$
\left|\int_{\mathbf{R}^{3}}\left(m_{\Lambda}-\rho_{\Lambda}\right) h_{\Lambda}\right| \leqslant C D\left(m_{\Lambda}-\rho_{\Lambda}, m_{\Lambda}-\rho_{\Lambda}\right)^{1 / 2}\left\|\nabla h_{\Lambda}\right\|_{L^{2}\left(\mathbf{R}^{3}\right)},
$$

(this is easy to check using Fourier transforms), where here and below $C$ denotes a positive constant that is independent of $\Lambda$. 
Let us begin with the proof of Lemma 4.3. In view of the expression (70) along with the bound from below (73) and the bound from above for the energy (68), we deduce that the density of a minimizer satisfies

$$
\frac{1}{|\Lambda|} D\left(m_{\Lambda}-\rho_{\Lambda}, m_{\Lambda}-\rho_{\Lambda}\right) \leqslant C
$$

Next, it only remains to apply (79) with a special choice for $h_{\Lambda}$; namely, $h_{\Lambda}$ is such that $0 \leqslant h_{\Lambda} \leqslant 1, h_{\Lambda} \equiv 1$ on $\Gamma(\Lambda), h_{\Lambda}=0$ on $\left\{x \in \Gamma(\Lambda)^{c} ; d(x ; \partial \Gamma(\Lambda)) \geqslant 1\right\}$, and $\int_{\mathbf{R}^{3}}\left|\nabla h_{\Lambda}\right|^{2}=\mathrm{o}(|\Lambda|)$ (see the details in Chapter 3 of [11]).

In order to prove Lemma 4.4, we remark that (76) implies in particular

$$
\int_{\mathbf{R}^{3}}\left|\nabla \sqrt{\rho_{\Lambda}}\right|^{2} \leqslant C|\Lambda|
$$

Then, we may apply the inequality (79) with $h_{\Lambda}=\sqrt{\rho_{\Lambda}}$, and we deduce from (80) and (81) that

$$
\left|\int_{\mathbf{R}^{3}}\left(m_{\Lambda}-\rho_{\Lambda}\right) \sqrt{\rho_{\Lambda}}\right| \leqslant C|\Lambda|
$$

The Cauchy-Schwarz inequality now gives

$$
\left|\int_{\mathbf{R}^{3}} m_{\Lambda} \sqrt{\rho_{\Lambda}}\right| \leqslant\left\|m_{\Lambda}\right\|_{L^{2}\left(\mathbf{R}^{3}\right)}\left\|\sqrt{\rho_{\Lambda}}\right\|_{L^{2}\left(\mathbf{R}^{3}\right)} \leqslant C|\Lambda|
$$

The same argument carries through to the case of point nuclei, replacing $m_{\Lambda}$ by $\chi_{\Gamma(\Lambda)}$ everywhere above, since the various bounds obtained in the course of the proof of Lemma 4.2 yield in particular $D\left(\chi_{\Gamma(\Lambda)}-\rho_{\Lambda}, \chi_{\Gamma(\Lambda)}-\rho_{\Lambda}\right) \leqslant C|\Lambda|$.

\subsection{A striking example}

In view of the estimates of the previous section, it seems reasonable to believe that the Hartree energy per unit volume admits a thermodynamic limit (though it is not explicitly proven). The natural question is then to determine even formally such a limit. We go back to the expression for the Hartree energy given in (64), that is

$$
E_{\Lambda}^{H}\left(\varphi_{1} ; \ldots ; \varphi_{|\Lambda|}\right)=\sum_{i=1}^{|\Lambda|}\left(\int_{\mathbf{R}^{3}}\left|\nabla \varphi_{i}\right|^{2}-\frac{1}{2} D\left(\left|\varphi_{i}\right|^{2},\left|\varphi_{i}\right|^{2}\right)\right)-\int_{\mathbf{R}^{3}} V_{\Lambda} \rho+\frac{1}{2} D(\rho, \rho),
$$

that can be rewritten in the usual way

$$
E_{\Lambda}^{H}\left(\varphi_{1} ; \ldots ; \varphi_{|\Lambda|}\right)=\sum_{i=1}^{|\Lambda|} \int_{\mathbf{R}^{3}}\left|\nabla \varphi_{i}\right|^{2}-\int_{\mathbf{R}^{3}} V_{\Lambda} \rho+\frac{1}{2} \sum_{1 \leqslant i \neq j \leqslant|\Lambda|} D\left(\left|\varphi_{i}\right|^{2},\left|\varphi_{j}\right|^{2}\right) .
$$


It is then tempting to argue as follows: in view of the large number $|\Lambda|$ of functions asymptotically involved, there should not be a large difference between the above energy and the restricted Hartree energy given in (45). In other words, since the number of "diagonal" terms $[i=j]$, namely $|\Lambda|$, is small compared to the numbers $\left(|\Lambda|^{2}-|\Lambda|\right)$ of the "off-diagonal" terms $[i \neq j]$, one could be tempted to replace the sum $\sum_{1 \leqslant i \neq j \leqslant|\Lambda|}$ by the sum $\sum_{1 \leqslant i, j \leqslant|\Lambda|}$, and obtain the same problem in the thermodynamic limit, both for the Hartree and the restricted Hartree problem. As a consequence, the Hartree model should degenerate in the thermodynamic limit, to a periodic problem of density functional type. As we shall see, this guess is wrong. We shall provide two arguments in the favor of this claim. We shall first prove, for a very special case detailed just below, that the thermodynamic limit of the energy per unit volume converges to the periodic Hartree model we have set before. Secondly, we prove in Section 4.4 below that the upper limit of the energy per unit volume in the general Hartree setting may be bounded from above by the periodic Hartree model, provided the unit cell of the crystal is a cube, and provided there exists a minimizer of the Hartree periodic model which shares the symmetries of the unit cube. In both cases, the limit of the energy per unit volume is clearly strictly smaller than the corresponding periodic energy in the restricted Hartree setting, as shown in Section 4.4 below.

To convince the reader that the diagonal terms do play a role even in the limit, we consider the Hartree model for smeared out nuclei, and we moreover choose a very particular form of nuclei. Let us denote by $\varphi_{C}$ a positive minimizer of the Choquard problem introduced in (65) (there are many minimizers, all equal from one another up to a translation, we just pick out one of them). We choose $\varphi_{C}^{2}$ as a shape of the nuclei. For each finite set $\Lambda$, the measure $m_{\Lambda}$ defining the density of nuclei is therefore

$$
m_{\Lambda}=\sum_{k \in \Lambda} \varphi_{C}^{2}(\cdot-k)
$$

The Hartree energy (70) then reads

$$
\begin{aligned}
E_{\Lambda}^{m, H}\left(\varphi_{1} ; \ldots ; \varphi_{|\Lambda|}\right)= & \sum_{i=1}^{|\Lambda|}\left[\int_{\mathbf{R}^{3}}\left|\nabla \varphi_{i}\right|^{2}-\frac{1}{2} D\left(\varphi_{i}^{2}, \varphi_{i}^{2}\right)\right] \\
& +\frac{1}{2} D\left(m_{\Lambda}-\rho_{\Lambda}, m_{\Lambda}-\rho_{\Lambda}\right)-\frac{1}{2}|\Lambda| D\left(\varphi_{C}^{2}, \varphi_{C}^{2}\right) .
\end{aligned}
$$

It is straightforward to see on the above expression that any $\left(\varphi_{1} ; \ldots ; \varphi_{|\Lambda|}\right)$ such that $\left\{\varphi_{i} ; 1 \leqslant i \leqslant|\Lambda|\right\}=\left\{\varphi_{C}(\cdot-k) ; k \in \Lambda\right\}$ defines a minimizer of this Hartree problem. Indeed, by definition of the Choquard minimum $\varphi_{C}$ we have, for all arbitrary function $\varphi$

$$
\int_{\mathbf{R}^{3}}|\nabla \varphi|^{2}-\frac{1}{2} D\left(\varphi^{2}, \varphi^{2}\right) \geqslant \int_{\mathbf{R}^{3}}\left|\nabla \varphi_{C}\right|^{2}-\frac{1}{2} D\left(\varphi_{C}^{2}, \varphi_{C}^{2}\right)
$$

with equality if and only if $\varphi=\varphi_{C}(\cdot+y)$, for some $y$ in $\mathbf{R}^{3}$, and on the other hand

$$
\frac{1}{2} D\left(m_{\Lambda}-\rho_{\Lambda}, m_{\Lambda}-\rho_{\Lambda}\right) \geqslant 0,
$$


with equality when $m_{\Lambda}=\rho_{\Lambda}=\sum_{k \in \Lambda} \varphi_{k}^{2}$, which happens precisely when $\left\{\varphi_{k} ; 1 \leqslant k \leqslant\right.$ $|\Lambda|\}=\left\{\varphi_{C}(\cdot-k) ; k \in \Lambda\right\}$.

As a consequence, we have in this setting

$$
\frac{1}{|\Lambda|} I_{\Lambda}^{m, H}=I_{C}-\frac{1}{2} D\left(\varphi_{C}^{2}, \varphi_{C}^{2}\right),
$$

and, also,

$$
\begin{gathered}
\lim _{\Lambda \rightarrow \infty} \frac{I_{\Lambda}^{m, H}}{|\Lambda|}=\inf \left\{\int_{\mathbf{R}^{3}}|\nabla \varphi|^{2}-\frac{1}{2} D\left(\varphi^{2}, \varphi^{2}\right)+\frac{1}{2} D_{G}(m-\rho, m-\rho)-\frac{1}{2} D(m, m) ;\right. \\
\left.\varphi \in H^{1}\left(\mathbf{R}^{3}\right), \int_{\mathbf{R}^{3}} \varphi^{2}=1, \rho=\sum_{k \in \mathbf{Z}^{3}}|\varphi|^{2}(\cdot+k)\right\} .
\end{gathered}
$$

We have therefore proven

PROPOSITION 4.1. - In the special case when the shape of the nuclei is given by the Choquard minimizer $\varphi_{C}$ through (82), the energy per unit volume for the Hartree model converges in the thermodynamic limit to the infimum of the associated periodic problem (36)-(37)-(32), up to the usual additive constant $M / 2$.

In addition, the Hartree minimizer, which in this special case is $\left(\varphi_{C}(\cdot-k)\right)_{k \in \Lambda}$ converges to $\left(\varphi_{C}(\cdot-k)\right)_{k \in \mathbf{Z}^{3}}$, where $\varphi_{C}$ minimizes (36)-(37)-(32).

The above setting has therefore allowed us to figure one possible thermodynamic limit for the generic Hartree model. We are now going to study this periodic problem.

\subsection{Well-posedness of the Hartree periodic problem}

In view of the above example, we believe that we are founded to consider the periodic Hartree problem introduced in (30)-(31) and that we recall here for convenience

$$
\begin{gathered}
I_{p e r}^{H}=\inf \left\{E_{p e r}^{H}(\varphi) ; \varphi \in H^{1}\left(\mathbf{R}^{3}\right), \int_{\mathbf{R}^{3}}|\varphi|^{2}=1\right\}, \\
E_{p e r}^{H}(\varphi)=\int_{\mathbf{R}^{3}}|\nabla \varphi|^{2}-\frac{1}{2} D\left(|\varphi|^{2},|\varphi|^{2}\right)-\int_{Q} G \rho+\frac{1}{2} D_{G}(\rho, \rho),
\end{gathered}
$$

with

$$
\rho(x)=\sum_{k \in \mathbf{Z}^{3}}|\varphi|^{2}(x+k) .
$$

Note that (83) may equivalently be written as

$$
E_{p e r}^{H}(\varphi)=\int_{\mathbf{R}^{3}}|\nabla \varphi|^{2}-\frac{1}{2} D\left(|\varphi|^{2},|\varphi|^{2}\right)-\int_{\mathbf{R}^{3}} G \varphi^{2}+\frac{1}{2} \iint_{\mathbf{R}^{3} \times \mathbf{R}^{3}} \varphi^{2}(x) G(x-y) \varphi^{2}(y) \mathrm{d} x \mathrm{~d} y .
$$


The analogous problem for smeared nuclei is given by

$$
I_{p e r}^{m, H}=\inf \left\{E_{p e r}^{m, H}(\varphi) ; \varphi \in H^{1}\left(\mathbf{R}^{3}\right), \int_{\mathbf{R}^{3}}|\varphi|^{2}=1\right\},
$$

where the periodic energy $E_{p e r}^{m, H}$ is defined as follows

$$
\begin{aligned}
E_{p e r}^{m, H}(\varphi)= & \int_{\mathbf{R}^{3}}|\nabla \varphi|^{2}-\frac{1}{2} D\left(|\varphi|^{2},|\varphi|^{2}\right) \\
& +\frac{1}{2} D_{G}(\rho-m, \rho-m)-\frac{1}{2} D_{G}(m, m) .
\end{aligned}
$$

We have the following result announced in Section 2

THEOREM 2.2 (Well-posedness of the $\mathrm{H}$ periodic problem). - The minimization problem defined by (30)-(31) (respectively by (36)-(37)) admits a minimum. In addition, any minimizing sequence of (30)-(31) (respectively (36)-(37)) is relatively compact in $H^{1}\left(\mathbf{R}^{3}\right)$, up to a translation.

The rest of this paragraph is devoted to the proof of this theorem. For the sake of clarity, we only do the proof in the smeared nuclei case. A straightforward adaptation of the following arguments allows one to conclude in the case of point nuclei.

Proof of Theorem 2.2. - Let us first make the following observation. Since the quadratic form $f \mapsto D_{G}(f, f)$ is non-negative, we may use, once more the comparison from below by the Choquard energy, and we obtain without difficulty that $I_{p e r}^{m, H}>-\infty$.

Step 1: Compactness of the periodic density.

Let $\varphi_{n}$ be a minimizing sequence of (85)-(86). It is clear that the Choquard energy

$$
\int_{\mathbf{R}^{3}}\left|\nabla \varphi_{n}\right|^{2}-\frac{1}{2} D\left(\left|\varphi_{n}\right|^{2},\left|\varphi_{n}\right|^{2}\right)
$$

of $\varphi_{n}$ is bounded, and therefore, by using (67), that $\varphi_{n}$ is bounded in $H^{1}\left(\mathbf{R}^{3}\right)$. Consequently, $\rho_{n}=\sum_{k \in \mathbf{Z}^{3}} \varphi_{n}^{2}(\cdot-k)$ satisfies

$$
\sqrt{\rho}_{n} \text { is bounded in } H_{p e r}^{1}(Q),
$$

for

$$
\int_{Q} \rho_{n}=\int_{\mathbf{R}^{3}} \varphi_{n}^{2}=1
$$

and by convexity

$$
\int_{Q}\left|\nabla \sqrt{\rho}_{n}\right|^{2} \leqslant \int_{\mathbf{R}^{3}}\left|\nabla \varphi_{n}\right|^{2} .
$$

It follows that (extracting a subsequence if necessary) $\sqrt{\rho}_{n}$ converges weakly in $H_{p e r}^{1}(Q)$, strongly in $L^{p}(Q), 2 \leqslant p<6$ (by the Rellich-Kondrakov theorem), and thus 
almost everywhere on $\mathbf{R}^{3}$, to some $\sqrt{\rho}$ satisfying

$$
\int_{Q} \rho=1,
$$

and

$$
\lim _{n \rightarrow+\infty} D_{G}\left(\rho_{n}-m, \rho_{n}-m\right)=D_{G}(\rho-m, \rho-m) .
$$

The "only" question that remains to settle is the behaviour of $\varphi_{n}$ itself in order to pass to the (lower) limit in the Choquard energy (87). (Note that the weak convergence of $\varphi_{n}$ in $H^{1}\left(\mathbf{R}^{3}\right)$ is not sufficient to conclude.) Another point is worth to be noticed at this stage. Since $m$ and $G$ are periodic, we obviously check that, for any $k$ in $\mathbf{Z}^{3}$ and $\varphi$ in $H^{1}\left(\mathbf{R}^{3}\right), E_{p e r}^{m, H}(\varphi(\cdot+k))=E_{p e r}^{m, H}(\varphi)$, and that $\varphi(\cdot+k)$ and $\varphi$ yield the same density $\rho$ through (84). Therefore, the minimization problem under consideration has some translation invariance, and the meaningful notion of convergence for the minimizing sequences is the convergence up to some translation. For these reasons, we shall adopt in the following the concentration-compactness approach (and its terminology), for which we refer the reader to [34] and the Appendix of [35].

Step 2: Vanishing does not occur.

We argue by contradiction, and begin by assuming that the sequence $\varphi_{n}$ vanishes, that is to say, for all $R>0$,

$$
\lim _{n \rightarrow+\infty} \sup _{x \in \mathbf{R}^{3}} \int_{x+B_{R}} \varphi_{n}^{2}=0 .
$$

A standard consequence of (93) (see [34]) is that $\varphi_{n}$ converges to 0 in $L^{p}\left(\mathbf{R}^{3}\right)$ for all $2<p<6$. Hence,

$$
\lim _{n \rightarrow+\infty} D\left(\varphi_{n}^{2}, \varphi_{n}^{2}\right)=0
$$

On the other hand, by the convexity argument used in (90), we have

$$
\liminf _{n \rightarrow+\infty} \int_{\mathbf{R}^{3}}\left|\nabla \varphi_{n}\right|^{2} \geqslant \liminf _{n \rightarrow+\infty} \int_{Q}|\nabla \sqrt{\rho}|^{2} \geqslant \int_{Q}|\nabla \sqrt{\rho}|^{2},
$$

where we recall that $\rho$ is the limit of $\rho_{n}$. It follows from (94) and (95) that

$$
\begin{aligned}
I_{p e r}^{m, H}+\frac{1}{2} D_{G}(m, m) & =\liminf _{n \rightarrow+\infty}\left[\int_{\mathbf{R}^{3}}\left|\nabla \varphi_{n}\right|^{2}-\frac{1}{2} D\left(\varphi_{n}^{2}, \varphi_{n}^{2}\right)+\frac{1}{2} D_{G}\left(\rho_{n}-m, \rho_{n}-m\right)\right] \\
& \geqslant \int_{Q}|\nabla \sqrt{\rho}|^{2}+\frac{1}{2} D_{G}(\rho-m, \rho-m) .
\end{aligned}
$$

Therefore, contradicting the vanishing assumption amounts to exhibiting some $\varphi \in$ $H^{1}\left(\mathbf{R}^{3}\right)$ such that the following two properties are satisfied

$$
\sum_{k \in \mathbf{Z}^{3}} \varphi^{2}(\cdot-k)=\rho
$$


and

$$
\int_{\mathbf{R}^{3}}|\nabla \varphi|^{2}-\frac{1}{2} D\left(\varphi^{2}, \varphi^{2}\right)<\int_{Q}|\nabla \sqrt{\rho}|^{2} .
$$

Indeed, assume for a moment that we have at our disposal some $\varphi$ satisfying (97)-(98). We then have

$$
\begin{aligned}
& I_{p e r}^{m, H}+\frac{1}{2} D_{G}(m, m) \\
& \leqslant \inf \left\{\int_{\mathbf{R}^{3}}|\nabla \psi|^{2}-\frac{1}{2} D\left(\psi^{2}, \psi^{2}\right) ; \psi \in H^{1}\left(\mathbf{R}^{3}\right), \sum_{k \in \mathbf{Z}^{3}} \psi^{2}(\cdot-k)=\rho\right\} \\
& \quad+\frac{1}{2} D_{G}(\rho-m, \rho-m) \\
& \leqslant \int_{\mathbf{R}^{3}}|\nabla \varphi|^{2}-\frac{1}{2} D\left(\varphi^{2}, \varphi^{2}\right)+\frac{1}{2} D_{G}(\rho-m, \rho-m) \\
&<\int_{Q}|\nabla \sqrt{\rho}|^{2}+\frac{1}{2} D_{G}(\rho-m, \rho-m) .
\end{aligned}
$$

This contradicts (96).

In order to construct a convenient $\varphi$, let us first of all consider a partition of unity. We fix some $\omega_{1} \in \mathcal{D}\left(\mathbf{R}^{3}\right), \omega_{1} \geqslant 0, \sqrt{\omega}_{1} \in W^{1, \infty}\left(\mathbf{R}^{3}\right)$, and

$$
\sum_{k \in \mathbf{Z}^{3}} \omega_{1}(\cdot-k)=1
$$

(Such a function exists; indeed, for any $\varphi \in \mathcal{D}\left(\mathbf{R}^{3}\right)$, with $\varphi \geqslant 0$ and $\int \varphi=1, \varphi \star \chi_{Q}$ provides an example.) We next scale this function $\omega_{1}$ by defining, for all $n \in \mathbf{N}$,

$$
\omega_{n}=\frac{1}{n^{3}} \omega_{1}\left(\frac{\cdot}{n}\right) .
$$

The function $\omega_{n}$ yields again a partition of unity

$$
\sum_{k \in \mathbf{Z}^{3}} \omega_{n}(\cdot-k)=1
$$

Indeed, it suffices to remark that the function in the left-hand side of (99) is periodic and to show by a simple calculation, that we leave to the reader, that its Fourier transform at the points of $\mathbf{Z}^{3}$ is everywhere zero except at 0 where its takes the value one.

Next, we consider the sequence of functions $\psi_{n}=\sqrt{\rho} \sqrt{\omega}_{n}$. It is easily checked that $\psi_{n} \in H^{1}\left(\mathbf{R}^{3}\right)$ when $\sqrt{\rho} \in H_{p e r}^{1}(Q)$. By construction,

$$
\sum_{k \in \mathbf{Z}^{3}} \psi_{n}^{2}(\cdot-k)=\rho(\cdot) \sum_{k \in \mathbf{Z}^{3}} \omega_{n}(\cdot-k)=\rho(\cdot),
$$


and we shall prove that, for $n$ large enough,

$$
\int_{\mathbf{R}^{3}}\left|\nabla \psi_{n}\right|^{2}-\frac{1}{2} D\left(\psi_{n}^{2}, \psi_{n}^{2}\right)<\int_{Q}|\nabla \sqrt{\rho}|^{2} .
$$

We claim that

$$
\begin{aligned}
D\left(\psi_{n}^{2}, \psi_{n}^{2}\right) & =\frac{1}{n} \iint_{\mathbf{R}^{3} \times \mathbf{R}^{3}} \frac{\rho(n x) \rho(n y) \omega_{1}(x) \omega_{1}(y)}{|x-y|} \mathrm{d} x \mathrm{~d} y \\
& =\frac{1}{n} D\left(\omega_{1}, \omega_{1}\right)+\mathrm{o}\left(\frac{1}{n}\right) .
\end{aligned}
$$

In order to prove this claim, let us remark first that, by the Riemann-Lebesgue theorem, the sequence of functions $\rho(n \cdot)$ converges weakly in $L_{l o c}^{p}$, for every $1 \leqslant p \leqslant 3$, to the constant function of value $\int_{Q} \rho=1$ as $n$ goes to infinity.

Denoting for a while $\rho^{n}(x)=\rho(n x)$, we remark next that

$$
\iint_{\mathbf{R}^{3} \times \mathbf{R}^{3}} \frac{\rho(n x) \rho(n y) \omega_{1}(x) \omega_{1}(y)}{|x-y|} \mathrm{d} x \mathrm{~d} y=\int_{\mathbf{R}^{3}}\left(\rho^{n} \omega_{1} \star \frac{1}{|x|}\right) \rho^{n} \omega_{1} .
$$

It is easy to see that $\left(\rho^{n} \omega_{1}\right) \star \frac{1}{|x|}$ is bounded in $H_{l o c}^{2}\left(\mathbf{R}^{3}\right)$ since $\rho^{n} \omega_{1}$ is bounded in $L^{2}\left(\mathbf{R}^{3}\right)$. Therefore it strongly converges in $L_{\text {loc }}^{2}\left(\mathbf{R}^{3}\right)$, to $\omega_{1} \star \frac{1}{|x|}$, and thus $\left(\left(\rho^{n} \omega_{1}\right) \star \frac{1}{|x|}\right) \omega_{1}$ converges in $L^{2}\left(\mathbf{R}^{3}\right)$ to $\left(\omega_{1} \star \frac{1}{|x|}\right) \omega_{1}$. As $\rho^{n}$ converges weakly locally in $L^{2}$ to 1 , we obtain (100).

We now estimate

$$
\begin{aligned}
\int_{\mathbf{R}^{3}}\left|\nabla \psi_{n}\right|^{2} & =\int_{\mathbf{R}^{3}}|\nabla \sqrt{\rho}|^{2} \omega_{n}+2 \int_{\mathbf{R}^{3}} \sqrt{\rho} \sqrt{\omega}_{n} \nabla \sqrt{\rho} \cdot \nabla \sqrt{\omega_{n}}+\int_{\mathbf{R}^{3}}\left|\nabla \sqrt{\omega_{n}}\right|^{2} \rho \\
& =\int_{Q}|\nabla \sqrt{\rho}|^{2}+\frac{1}{2 n} \int_{\mathbf{R}^{3}} \nabla \rho(n x) \cdot \nabla \omega_{1}(x) \mathrm{d} x+\frac{1}{n^{2}} \int_{\mathbf{R}^{3}}\left|\nabla \sqrt{\omega_{1}}(x)\right|^{2} \rho(n x) \mathrm{d} x .
\end{aligned}
$$

To treat the last term, we remark that

$$
\begin{aligned}
\frac{1}{n^{2}} \int_{\mathbf{R}^{3}}\left|\nabla \sqrt{\omega_{1}}(x)\right|^{2} \rho(n x) \mathrm{d} x & \leqslant \frac{1}{n^{2}} \int_{\operatorname{Supp}\left(\omega_{1}\right)}\left|\nabla \sqrt{\omega_{1}}(x)\right|^{2} \rho(n x) \mathrm{d} x \\
& \leqslant \frac{1}{n^{2}}\left\|\nabla \sqrt{\omega_{1}}\right\|_{L^{\infty}}^{2} \int_{\operatorname{Supp}\left(\omega_{1}\right)} \rho(n x) \mathrm{d} x \\
& \leqslant \frac{1}{n^{2}} C^{t e}\left\|\nabla \sqrt{\omega}_{1}\right\|_{L^{\infty}}^{2} \int_{Q} \rho=\mathrm{O}\left(\frac{1}{n^{2}}\right) .
\end{aligned}
$$

For the second term, we compute by Green's formula

$$
\int_{\mathbf{R}^{3}} \nabla \rho(n x) \cdot \nabla \omega_{1}(x) \mathrm{d} x=-\int_{\mathbf{R}^{3}} \rho(n x) \Delta \omega_{1}(x) \mathrm{d} x
$$


and the right-hand side goes to 0 as $n$ goes to infinity, for $\rho(n x)$ converges to 1 in $\mathcal{D}^{\prime}\left(\mathbf{R}^{3}\right)$, and $\int_{\mathbf{R}^{3}} \Delta \omega_{1}=0$. Therefore, we have

$$
\frac{1}{2 n} \int_{\mathbf{R}^{3}} \nabla \rho(n x) \cdot \nabla \omega_{1}(x) \mathrm{d} x=\mathrm{o}\left(\frac{1}{n}\right) .
$$

Collecting these informations, we deduce

$$
\int_{\mathbf{R}^{3}}\left|\nabla \psi_{n}\right|^{2}=\int_{Q}|\nabla \sqrt{\rho}|^{2}+\mathrm{o}\left(\frac{1}{n}\right) .
$$

From (101) together with (100), we obtain

$$
\int_{\mathbf{R}^{3}}\left|\nabla \psi_{n}\right|^{2}-\frac{1}{2} D\left(\psi_{n}^{2}, \psi_{n}^{2}\right)=\int_{Q}|\nabla \sqrt{\rho}|^{2}-\frac{1}{2 n} D\left(\omega_{1}, \omega_{1}\right)+\mathrm{o}\left(\frac{1}{n}\right) .
$$

Therefore, for $n$ large enough, we obtain a convenient function $\varphi$ in (97)-(98) by setting $\varphi=\psi_{n}$. We have thus reached a contradiction, and it follows that vanishing does not occur. The next step consists of ruling out dichotomy. As we shall see, this is somewhat more intricate than ruling out vanishing.

Step 3: Dichotomy does not occur.

Let us come back to the minimizing sequence $\varphi_{n}$ of our problem. Since (93) does not occur, there exist a subsequence of $\varphi_{n}$ (still denoted by $\varphi_{n}$ ), $R_{0}>0, \varepsilon_{0}>0$, and a sequence of points $y_{n}$ in $\mathbf{R}^{3}$, such that

$$
\int_{B_{R_{0}}} \varphi_{n}^{2}\left(\cdot+y_{n}\right) \geqslant \varepsilon_{0} .
$$

By setting $y_{n}=\left[y_{n}\right]+k_{n}$, with $\left[y_{n}\right]$ in $Q$ and $k_{n}$ in $\mathbf{Z}^{3}$, and $\tilde{\varphi}_{n}=\varphi_{n}\left(\cdot+k_{n}\right)$, we obtain another minimizing sequence $\tilde{\varphi}_{n}$ of $I_{p e r}^{m, H}$, which yields the same limit density $\rho$. Moreover, since $\left[y_{n}\right]$ is bounded, we infer from (102) that the weak limit of $\tilde{\varphi}_{n}$ in $L^{2}\left(\mathbf{R}^{3}\right)$ is not identically zero. In all that follows, we shall work with the new sequence $\tilde{\varphi}_{n}$ (still denoted by $\varphi_{n}$ for simplicity). Without loss of generality, we may assume that $\varphi_{n} \geqslant 0$. Moreover, with the help of Ekeland's principle [16], given a minimizing sequence $\hat{\varphi}_{n}$, we may construct a new minimizing sequence $\varphi_{n}$, such that

$$
I_{p e r}^{m, H} \leqslant E_{p e r}^{m, H}\left(\varphi_{n}\right) \leqslant E_{p e r}^{m, H}\left(\hat{\varphi}_{n}\right),
$$

and that

$$
\left\|\hat{\varphi}_{n}-\varphi_{n}\right\|_{L^{2}\left(\mathbf{R}^{3}\right)} \rightarrow 0, \quad \text { as } n \rightarrow \infty .
$$

In particular, it is easily seen that the densities corresponding to $\varphi_{n}$ and $\hat{\varphi}_{n}$, namely $\rho_{n}=\sum_{k \in \mathbf{Z}^{3}} \varphi_{n}^{2}(\cdot-k)$ and $\hat{\rho}_{n}=\sum_{k \in \mathbf{Z}^{3}} \hat{\varphi}_{n}^{2}(\cdot-k)$, converge to the same limit $\rho$. Moreover, $\varphi_{n}$ satisfies 


$$
-\Delta \varphi_{n}-\left(\varphi_{n}^{2} \star \frac{1}{|x|}\right) \varphi_{n}+\left(G \star_{Q}\left(\rho_{n}-m\right)\right) \varphi_{n}+\varepsilon_{n} \varphi_{n} \rightarrow 0,
$$

in $L^{2}\left(\mathbf{R}^{3}\right)$ (at least), as $n$ goes to infinity, for some real number $\varepsilon_{n}$. We recall that, in (105), $m$ denotes the measure defining the smeared nucleus in the unit cell $Q$, and we have denoted

$$
G \star_{Q}\left(\rho_{n}-m\right)=\int_{Q} G(x-y)\left(\rho_{n}(y)-m(y)\right) \mathrm{d} y .
$$

In addition, $\varepsilon_{n}$ is a sequence of "almost" Lagrange multipliers. Using the facts that all minimizing sequences of $I_{p e r}^{m, H}$ are bounded in $H^{1}\left(\mathbf{R}^{3}\right)$, we then easily deduce, from (104) and Sobolev and Hölder's inequalities, that

$$
\left\|\hat{\varphi}_{n}-\varphi_{n}\right\|_{L^{p}\left(\mathbf{R}^{3}\right)} \rightarrow 0, \quad \text { as } n \rightarrow \infty,
$$

for every $2 \leqslant p<6$, and we also check from (105) that $\varepsilon_{n}$ is bounded. Moreover, we may assume that $\varphi_{n}$ converges to $\varphi_{1} \geqslant 0$, weakly in $H^{1}\left(\mathbf{R}^{3}\right)$, strongly in $L_{l o c}^{p}\left(\mathbf{R}^{3}\right)$, for every $1 \leqslant p<6$, and almost everywhere on $\mathbf{R}^{3}$. Moreover, because of $(102), \int_{\mathbf{R}^{3}} \varphi_{1}^{2}>0$. Of course, if $\int_{\mathbf{R}^{3}} \varphi_{1}^{2}=1$, the proof is over. Indeed, (106) then yields the strong convergence of $\varphi_{n}$ and $\hat{\varphi}_{n}$ to $\varphi_{1}$ in $L^{p}\left(\mathbf{R}^{3}\right)$, for every $2 \leqslant p<6$. In particular, $\rho=\sum_{k \in \mathbf{Z}^{3}} \varphi_{1}^{2}(\cdot-k)$, and $\varphi_{n}^{2} \star \frac{1}{|x|}$ converges to $\varphi_{1}^{2} \star \frac{1}{|x|}$ for the strong convergence in $L^{q}\left(\mathbf{R}^{3}\right)$, for every $3<q<+\infty$. Hence,

$$
\lim _{n \rightarrow+\infty} D\left(\varphi_{n}^{2}, \varphi_{n}^{2}\right)=D\left(\varphi_{1}^{2}, \varphi_{1}^{2}\right) .
$$

Using (92), we thus prove without difficulty that

$$
\begin{aligned}
I_{p e r}^{m, H} & =\limsup _{n \rightarrow+\infty} E_{p e r}^{m, H}\left(\hat{\varphi}_{n}\right) \\
& \geqslant \liminf _{n \rightarrow+\infty} E_{p e r}^{m, H}\left(\hat{\varphi}_{n}\right) \\
& \geqslant \liminf _{n \rightarrow+\infty} E_{p e r}^{m, H}\left(\varphi_{n}\right) \\
& \geqslant E_{p e r}^{m, H}\left(\varphi_{1}\right) \\
& \geqslant I_{p e r}^{m, H} .
\end{aligned}
$$

Therefore, all above inequalities are equalities. In particular, $\varphi_{1}$ is a minimizer of $I_{p e r}^{m, H}$. Moreover, we deduce from (107) and (92) that

$$
\lim _{n \rightarrow+\infty} \int_{\mathbf{R}^{3}}\left|\nabla \hat{\varphi}_{n}\right|^{2}=\lim _{n \rightarrow+\infty} \int_{\mathbf{R}^{3}}\left|\nabla \varphi_{n}\right|^{2}=\int_{\mathbf{R}^{3}}\left|\nabla \varphi_{1}\right|^{2},
$$

and, thus, $\hat{\varphi}_{n}$ and $\varphi_{n}$ both converge to $\varphi_{1}$ for the strong convergence in $H^{1}\left(\mathbf{R}^{3}\right)$ (at least).

We now assume by contradiction that $\int_{\mathbf{R}^{3}} \varphi_{1}^{2}<1$, and set $\varphi_{2, n}=\varphi_{n}-\varphi_{1}$. We shall analyze the behaviour of the sequence $\varphi_{2, n}$, following the scheme of proof which is given in the Appendix of Lions [35]. Since $\varphi_{2, n}$ is bounded in $H^{1}\left(\mathbf{R}^{3}\right)$, we may reproduce with $\varphi_{2, n}$ the same argument as the one we just made on $\varphi_{n}$. We leave apart for the 
moment the case when $\varphi_{2, n}$ vanishes in the sense of Step 2 (this case will be ruled out in Step 4 below), and concentrate first on the most difficult case when a 'standard' dichotomy arises. That is to say, we assume that there exists a sequence of points $y_{n}$ in $\mathbf{R}^{3}$, with $\left|y_{n}\right| \rightarrow+\infty$, such that $\varphi_{2, n}\left(\cdot+y_{n}\right)$ converges to $\varphi_{2} \geqslant 0, \varphi_{2} \not \equiv 0$, weakly in $H^{1}\left(\mathbf{R}^{3}\right)$, strongly in $L_{l o c}^{p}\left(\mathbf{R}^{3}\right)$, for every $1 \leqslant p<6$, and almost everywhere on $\mathbf{R}^{3}$. When dichotomy occurs, we see, by reproducing several times the above argument, that the sequence $\varphi_{n}$ splits into many pieces, each piece going far away from all the others. Assuming that, at each step, the case of vanishing is left apart for a while, we infer the existence of functions $\varphi_{k} \in H^{1}\left(\mathbf{R}^{3}\right), \varphi_{k} \geqslant 0$, such that

$$
\varphi_{n}-\sum_{k=1}^{K} \varphi_{k}\left(\cdot+y_{k}^{(n)}\right) \rightarrow 0,
$$

strongly in $L^{2}\left(\mathbf{R}^{3}\right)$, as $n$ goes to infinity, and for some sequences of points $y_{k}^{(n)} \in \mathbf{R}^{3}$ such that $\left|y_{k}^{(n)}-y_{l}^{(n)}\right| \rightarrow+\infty$, if $k \neq l$. Let us observe that in (108) above, $2 \leqslant K \leqslant+\infty$ (when $K=1, \varphi_{n}$ is compact up to a translation). Our first step will consist in showing that $K$ is finite; that is to say, dichotomy may involve only finitely many pieces going far away from each over. Indeed, otherwise, by setting $\alpha_{k}=\int \varphi_{k}^{2}>0$, we must have $\alpha_{k}$ going to 0 , as $k$ goes to infinity, since $\sum_{k \geqslant 1} \alpha_{k}=1$, due to (108). Therefore, passing to the limit in (105) as $n$ goes to infinity, it is not difficult to check that the $\varphi_{k}$ 's are infinitely many (non-negative) solutions, in the sense of distributions at least, to

$$
-\Delta \varphi_{k}-\left(\varphi_{k}^{2} \star \frac{1}{|x|}\right) \varphi_{k}+W \varphi_{k}+\varepsilon \varphi_{k}=0
$$

such that $\varphi_{k}$ goes to 0 in $L^{2}\left(\mathbf{R}^{3}\right)$, as $k$ goes to infinity, where we have denoted

$$
W=G \star_{Q}(\rho-m),
$$

and where $\varepsilon$ is the limit of the "almost Lagrange multipliers" $\varepsilon_{n}$ appearing in (105). The two main points in (109) are that (a) the Lagrange multiplier $\varepsilon$ is the same in all the equations, which is a standard fact in the concentration-compactness approach, (b) the periodic potential $W$ is also the same, which is a consequence of the fact that the sequence of densities $\rho_{n}$ is known to be compact from Step 1. It is to be noticed that the argument which is used in the Appendix of [35] to prove that $K$ is finite, within some specific examples, is not valid in our case, for $W$ is periodic (in particular, it does not decay to 0 at infinity). Nevertheless, we may argue in the following way. Using (67) and the fact that $W$ is in $L_{u n i f}^{3 / 2}\left(\mathbf{R}^{3}\right)$, it is a standard exercise to deduce from (109) (that we apply to $\varphi_{k}$, and then integrate over $\left.\mathbf{R}^{3}\right)$, that $\varphi_{k}$ is bounded in $H^{1}\left(\mathbf{R}^{3}\right)$. Therefore, $\varphi_{k}$ converges to 0 strongly in $L^{p}\left(\mathbf{R}^{3}\right)$, for every $2 \leqslant p<6$, almost everywhere on $\mathbf{R}^{3}$, and weakly in $H^{1}\left(\mathbf{R}^{3}\right)$. In fact, going back to (109), we even deduce the strong convergence of $\varphi_{k}$ to 0 in $H^{1}\left(\mathbf{R}^{3}\right)$. We now claim that the sequence $v_{k}$ in $H^{1}\left(\mathbf{R}^{3}\right)$, which is defined by $v_{k}=\varphi_{k} /\left\|\varphi_{k}\right\|_{L^{2}\left(\mathbf{R}^{3}\right)}$, satisfies $\left\|v_{k}\right\|_{L^{2}\left(\mathbf{R}^{3}\right)}=1$, together with

$$
-\Delta v_{k}+W v_{k}+\varepsilon v_{k} \rightarrow 0, \quad \text { in } L^{2}\left(\mathbf{R}^{3}\right) .
$$


This is simply a consequence of the convergence of $\varphi_{k}$ to 0 , and of the well-known bound

$$
\left\|\varphi_{k}^{2} \star \frac{1}{|x|}\right\|_{L^{\infty}\left(\mathbf{R}^{3}\right)} \leqslant 2\left\|\varphi_{k}\right\|_{L^{2}\left(\mathbf{R}^{3}\right)}\left\|\nabla \varphi_{k}\right\|_{L^{2}\left(\mathbf{R}^{3}\right)}
$$

(which is, for example, deduced from Cauchy-Schwarz and Hardy's inequalities). These properties of $v_{k}$ imply that $-\varepsilon$ is in the spectrum of $-\Delta+W$ (see, for example, [43]). We then reach a contradiction, since we shall prove later that $-\varepsilon$ is actually strictly below the infimum of the spectrum of $-\Delta+W$ (see the comment following (126) below).

Having proved that dichotomy yields a finite number of "pieces", we may argue now, without loss of generality, as if there were only two pieces, $\varphi_{1}$, and $\varphi_{2, n}$, which is compact up to the translation along the vectors $y_{n}$. In other words, we have

$$
\varphi_{n}-\varphi_{1}-\varphi_{2}\left(\cdot-y_{n}\right) \rightarrow 0,
$$

strongly in $L^{p}\left(\mathbf{R}^{3}\right)$, for every $2 \leqslant p<6$, and weakly in $H^{1}\left(\mathbf{R}^{3}\right)$.

For reasons which will become clear in a moment, we define now the following family of minimization problems, indexed by a density $\tilde{\rho} \geqslant 0$, such that $\sqrt{\tilde{\rho}} \in H_{\text {per }}^{1}(Q)$

$$
I_{\tilde{\rho}}=\inf \left\{\int_{\mathbf{R}^{3}}|\nabla \varphi|^{2}-\frac{1}{2} D\left(\varphi^{2}, \varphi^{2}\right) ; \varphi \in H^{1}\left(\mathbf{R}^{3}\right), \sum_{k \in \mathbf{Z}^{3}} \varphi^{2}(x+k)=\tilde{\rho}(x)\right\} .
$$

The fact that the minimizing sequence $\varphi_{n}$ of the periodic Hartree problem (36) we consider has split into two pieces $\varphi_{1}$ and $\varphi_{2, n}$ (with the convergence (111)) while the density $\rho_{n}=\sum_{k \in \mathbf{Z}^{3}} \varphi_{n}^{2}(\cdot+k)$ converges to the periodic density $\rho$ of unit mass clearly implies that

$$
\left\{\begin{array}{l}
I_{\rho} \geqslant I_{\rho_{1}}+I_{\rho_{2}} \\
\rho=\rho_{1}+\rho_{2}
\end{array}\right.
$$

where we have denoted

$$
\rho_{i}=\sum_{k \in \mathbf{Z}^{3}} \varphi_{i}^{2}(\cdot+k), \quad i=1,2 .
$$

In addition, we necessarily have

$$
I_{\rho_{i}}=\int_{\mathbf{R}^{3}}\left|\nabla \varphi_{i}\right|^{2}-\frac{1}{2} D\left(\varphi_{i}^{2}, \varphi_{i}^{2}\right), \quad i=1,2,
$$

for we always have

$$
I_{\rho} \leqslant I_{\rho_{1}}+I_{\rho_{2}} .
$$

Indeed, let $\varepsilon>0$ be fixed, then there exist some functions $\psi_{i}, i=1,2$, in $\mathcal{D}\left(\mathbf{R}^{3}\right)$, such that $\sum_{k \in \mathbf{Z}^{3}} \psi_{i}^{2}(\cdot+k)=\rho_{i}$, and

$$
\int_{\mathbf{R}^{3}}\left|\nabla \psi_{i}\right|^{2}-\frac{1}{2} D\left(\psi_{i}^{2}, \psi_{i}^{2}\right) \leqslant I_{\rho_{i}}+\varepsilon
$$


Then, choosing $n$ in $\mathbf{Z}^{3}$, such that $\psi_{1}$ and $\psi_{2}(\cdot+n)$ have disjoint supports, and defining $\psi_{n}=\psi_{1}+\psi_{2}(\cdot+n)$, it is easily seen that $\sum_{k \in \mathbf{Z}^{3}} \psi_{n}^{2}(\cdot+k)=\rho_{1}+\rho_{2}=\rho$, and that

$$
\begin{aligned}
I_{\rho} & \leqslant \int_{\mathbf{R}^{3}}\left|\nabla \psi_{n}\right|^{2}-\frac{1}{2} D\left(\psi_{n}^{2}, \psi_{n}^{2}\right) \\
& \leqslant \sum_{i=1,2}\left[\int_{\mathbf{R}^{3}}\left|\nabla \psi_{i}\right|^{2}-\frac{1}{2} D\left(\psi_{i}^{2}, \psi_{i}^{2}\right)\right]+\mathrm{o}(1) \\
& \leqslant I_{\rho_{1}}+I_{\rho_{2}}+2 \varepsilon+\mathrm{o}(1),
\end{aligned}
$$

where o(1) goes to 0 when $n$ goes to infinity. Therefore, the inequality in (113) turns to be an inequality, which gives (115). We deduce (116), by letting $n$ go to infinity, and then $\varepsilon$ go to 0 in the above string of inequalities. In order to reach a contradiction with (113) (and therefore conclude that dichotomy does not occur), we shall now prove the converse inequality, namely

$$
I_{\rho}<I_{\rho_{1}}+I_{\rho_{2}}
$$

Note that these strict inequalities (118) involve variational problems with pointwise constraints, which is non-standard in the concentration-compactness method.

To proceed further, we need to obtain more information on the functions $\varphi_{1}$ and $\varphi_{2}$. In particular, we prove now that these functions have an exponential decay at infinity. Passing to the limit locally in (105), we thus obtain the system of equations

$$
\left\{\begin{array}{l}
-\Delta \varphi_{1}-\left(\varphi_{1}^{2} \star \frac{1}{|x|}\right) \varphi_{1}+W \varphi_{1}+\varepsilon \varphi_{1}=0, \\
-\Delta \varphi_{2}-\left(\varphi_{2}^{2} \star \frac{1}{|x|}\right) \varphi_{2}+W \varphi_{2}+\varepsilon \varphi_{2}=0,
\end{array}\right.
$$

with the periodic potential $W$ being defined by (110).

First of all, as $\varphi_{i} \geqslant 0$ and $\varphi_{i} \not \equiv 0$, it follows from (119) and from the Harnack inequality that $\varphi_{i}>0$. Next, we claim that

$$
\varepsilon>0 .
$$

For this purpose, we remark that $\int_{Q} \rho=1$, and therefore that

$$
\int_{Q} W=0 .
$$

A straightforward consequence of this observation is that the first eigenvalue of the operator $-\Delta+W$ on the unit cell $Q$, with periodic boundary conditions, denoted by $\lambda_{1}(-\Delta+W, p e r)$, is necessarily negative

$$
\lambda_{1}(-\Delta+W, \text { per })<0 .
$$

Indeed, it suffices to test the hamiltonian $-\Delta+W$ on the constant function $\frac{1}{|Q|}$, which yields $\lambda_{1}(-\Delta+W$, per $) \leqslant 0$, and to remark that this constant function cannot be the first eigenfunction of $-\Delta+W$ unless $W \equiv 0$. This latter case may happen, but it is even 
simpler to conclude that $\varepsilon>0$ then. Indeed, $W \equiv 0$ means $\rho=m$. The function $\varphi_{1}$ is then a positive solution to

$$
-\Delta \varphi_{1}-\left(\varphi_{1}^{2} \star \frac{1}{|x|}\right) \varphi_{1}+\varepsilon \varphi_{1}=0
$$

If $\varepsilon \leqslant 0$, we then obtain $\lambda_{1}\left(-\Delta-\varphi_{1}^{2} \star \frac{1}{|x|}, \Omega\right)>0$ on all bounded domain $\Omega$, and this cannot be true (use a rescaled function $u_{\sigma}(x)=\sigma^{3 / 2} u(\sigma x)$ with $\sigma$ small enough). We shall therefore assume that $W \not \equiv 0$ in the sequel, which implies (121).

We shall denote henceforth by $\varphi_{\text {per }}$ the first periodic eigenfunction of $-\Delta+W$ on $Q$.

From (121), we deduce that for any cube $K_{R}=[0, R]^{3}$ with $R$ large enough, the first eigenvalue of the operator $-\Delta+W$ on $K_{R}$ with homogeneous Dirichlet boundary conditions, denoted by $\lambda_{1}\left(-\Delta+W, K_{R}\right)$, is negative. Indeed, it suffices to take as a test function $\psi /\|\psi\|_{L^{2}}$ where $\psi$ is built as follows: $\psi$ is equal to $\varphi_{p e r}$ on $[1, R-1]^{3}$, and we glue to it a smooth function in order to satisfy the homogeneous Dirichlet boundary conditions on $\partial K_{R}$. Obviously,

$$
\int_{K_{R}}|\nabla \psi|^{2}+W \psi^{2}=(R-2)^{3} \lambda_{1}(-\Delta+W, \text { per })+\mathrm{O}\left(R^{2}\right),
$$

from where we deduce, for $R$ large enough,

$$
\lambda_{1}\left(-\Delta+W, K_{R}\right)<0,
$$

and, therefore a fortiori,

$$
\lambda_{1}\left(-\Delta-\left(\varphi_{1}^{2} \star \frac{1}{|x|}\right)+W, K_{R}\right)<0 .
$$

Let us now contradict (120) and assume that $\varepsilon \leqslant 0$. We then have

$$
\left\{\begin{array}{l}
-\Delta \varphi_{1}-\left(\varphi_{1}^{2} \star \frac{1}{|x|}\right) \varphi_{1}+W \varphi_{1}=-\varepsilon \varphi \geqslant 0 \\
\varphi_{1}>0
\end{array}\right.
$$

A standard argument, recalled in [11], shows that this implies that for all bounded domain $\Omega$, the first eigenvalue of the operator appearing in the left-hand side with homogeneous Dirichlet boundary conditions is non-negative:

$$
\lambda_{1}\left(-\Delta-\left(\varphi_{1}^{2} \star \frac{1}{|x|}\right)+W, \Omega\right) \geqslant 0 .
$$

Of course, we reach a contradiction with (122), and therefore we have proven our claim (120). 
Let us now see what this latter information implies on the behaviour at infinity of $\varphi_{1}$ (the same holding true of course for $\varphi_{2}$ respectively). We are in the following situation:

$$
\left\{\begin{array}{l}
-\Delta \varphi_{1}-\left(\varphi_{1}^{2} \star \frac{1}{|x|}\right) \varphi_{1}+W \varphi_{1}+\varepsilon \varphi_{1}=0 \\
\varphi_{1}>0, \\
W \text { is } Q \text {-periodic, } \quad \int_{Q} W=0, \quad \varepsilon>0 .
\end{array}\right.
$$

We claim that (124) implies that

$$
\varphi_{1}\left(\text { respectively } \varphi_{2}\right. \text { ) has an exponential decay at infinity. }
$$

This is probably a well-known fact. For the sake of consistency, we provide now one possible proof of this claim.

We begin with proving that (124) implies

$$
\lambda_{1}(-\Delta+W+\varepsilon, \text { per })>0 .
$$

Let us make some comment on this inequality. (126) implies that $-\varepsilon$ is strictly below the essential spectrum of the linearized operator $-\Delta+W$, since, as we are going to check, $\lambda_{1}(-\Delta+W$, per $)$ is precisely the bottom of the essential spectrum of $-\Delta+W$. Indeed, on the one hand, $-\Delta+W$ being a self-adjoint Schrödinger operators with a periodic potential, its spectrum consists only of essential spectrum and $\lambda_{1}(-\Delta+W$, per $)$ belongs to the spectrum (see $[43,15,52])$. On the other hand, since $W$ is in $L_{u n i f}^{p}\left(\mathbf{R}^{3}\right)$, for some $p>3 / 2$, it follows, from the Rayleigh-Ritz principle and Harnack's inequality, that the first eigenfunction of $-\Delta+W$ with periodic boundary conditions on $Q$ is positive. Therefore, $\lambda_{1}(-\Delta+W$,per $)$ is below the bottom of the spectrum of $-\Delta+W$ on $\mathbf{R}^{3}$ (see [46]). Let us turn now to the proof of (126).

It is easy to prove that $\lambda_{1}(-\Delta+W+\varepsilon, p e r)$ cannot be strictly negative. Indeed, should it be the case, we would just argue as we did before, building with the first eigenfunction of $-\Delta+W+\varepsilon$ (which is of course the function $\varphi_{\text {per }}$ introduced above) a convenient test function in order to prove that we then have necessarily $\lambda_{1}\left(-\Delta+W+\varepsilon, K_{R}\right)<0$ for some large enough cube $K_{R}$. Now, because of (124), we must have in particular (arguing as we did to prove (123)),

$$
\lambda_{1}(-\Delta+W+\varepsilon, \Omega)>0
$$

for any bounded domain $\Omega$, and we therefore reach a contradiction.

In order to prove (126), it still remains to show that $\lambda_{1}(-\Delta+W+\varepsilon$, per $)$ cannot be 0 either. For this purpose, we have to be a little more careful. If $\lambda_{1}(-\Delta+W+\varepsilon$, per $)=0$, we have

$$
(-\Delta+W+\varepsilon) \varphi_{\text {per }}=0 .
$$

We consider the function $\chi_{R} \varphi_{p e r}$, where $\chi_{R}$ is a cut-off function built as follows

$$
\chi_{R}(x)=\frac{1}{R^{3 / 2}} \chi_{1}\left(\frac{x}{R}\right),
$$


with $\chi_{1}$ a given smooth cut-off function, spherically symmetric, that vanishes outside the unit ball, and that is normalized by $\int \chi_{1}^{2}=1$. A simple computation shows that

$$
\int\left|\nabla\left(\chi_{R} \varphi_{p e r}\right)\right|^{2}=\int \varphi_{p e r}^{2}\left|\nabla \chi_{R}\right|^{2}-\int \chi_{R}^{2} \Delta \varphi_{p e r} \varphi_{p e r} .
$$

It follows that

$$
\begin{aligned}
& \int\left|\nabla\left(\chi_{R} \varphi_{p e r}\right)\right|^{2}-\left(\varphi_{1}^{2} \star \frac{1}{|x|}\right)\left(\chi_{R} \varphi_{p e r}\right)^{2}+(W+\varepsilon)\left(\chi_{R} \varphi_{p e r}\right)^{2} \\
& =\int \chi_{R}^{2}\left(-\Delta \varphi_{p e r}+(W+\varepsilon) \varphi_{p e r}\right) \varphi_{p e r}-D\left(\varphi_{1}^{2}, \chi_{R}^{2} \varphi_{p e r}^{2}\right)+\int \varphi_{p e r}^{2}\left|\nabla \chi_{R}\right|^{2} \\
& =-D\left(\varphi_{1}^{2}, \chi_{R}^{2} \varphi_{p e r}^{2}\right)+\int \varphi_{p e r}^{2}\left|\nabla \chi_{R}\right|^{2} .
\end{aligned}
$$

We remark that

$$
\int \varphi_{p e r}^{2}\left|\nabla \chi_{R}\right|^{2} \leqslant\left\|\varphi_{p e r}\right\|_{L^{\infty}}^{2} \int\left|\nabla \chi_{R}\right|^{2}=\mathrm{O}\left(\frac{1}{R^{2}}\right)
$$

and that

$$
D\left(\varphi_{1}^{2}, \chi_{R}^{2} \varphi_{\text {per }}^{2}\right) \geqslant\left(\inf \varphi_{\text {per }}\right)^{2} D\left(\varphi_{1}^{2}, \chi_{R}^{2}\right) \geqslant\left(\inf \varphi_{\text {per }}\right)^{2} \frac{1}{R} \int_{B_{R}} \varphi_{1}^{2}
$$

(the last inequality being true because of Newton's theorem). Inserting both informations into (127), we obtain that for $R$ large enough, the left-hand side of (127) is negative, and therefore

$$
\lambda_{1}\left(-\Delta-\varphi_{1}^{2} \star \frac{1}{|x|}+W+\varepsilon, B_{R}\right)<0
$$

which contradicts the fact that $\lambda_{1}\left(-\Delta-\varphi_{1}^{2} \star \frac{1}{|x|}+W+\varepsilon, \Omega\right) \geqslant 0$, for any bounded domain $\Omega$. Hence, (126) is proven.

As (126) holds, we may choose some $\varepsilon^{\prime}>0, \varepsilon^{\prime}<\varepsilon$, such that

$$
\lambda_{1}\left(-\Delta+W+\varepsilon^{\prime}, \text { per }\right)>0 .
$$

In addition, it is clear, using the fact that $\varphi_{1}^{2} \star \frac{1}{|x|}$ goes to zero at infinity, that for some $R$ large enough, (124) implies

$$
\left\{\begin{array}{l}
-\Delta \varphi_{1}+W \varphi_{1}+\varepsilon^{\prime} \varphi_{1} \leqslant 0, \quad \text { on } B_{R}^{c}, \\
\varphi_{1}>0, \\
W \text { is } Q \text {-periodic, } \quad \int_{Q} W=0, \quad \varepsilon^{\prime}>0 \\
\lambda_{1}\left(-\Delta+W+\varepsilon^{\prime}, \text { per }\right)>0 .
\end{array}\right.
$$

We are going to see that this implies the exponential decay of $\varphi_{1}$ at infinity. Let us first of all fix some $\theta \in] 0,1$ [ close enough to 1 , and some $\mu \in] 0, \varepsilon^{\prime}$ [ close enough to $\varepsilon^{\prime}$, such that

$$
\lambda_{1}\left(-\Delta+\frac{1}{\theta}(W+\mu), p e r\right)>0 .
$$


This is of course possible because of (128). We then introduce the corresponding periodic eigenfunction $\psi_{\text {per }}$ which satisfies

$$
\left\{\begin{array}{l}
-\Delta \psi_{p e r}+\frac{1}{\theta}(W+\mu) \psi_{p e r}>0 \quad \text { on } \mathbf{R}^{3}, \\
\psi_{p e r}>0, \\
\psi_{p e r} \text { is } Q \text {-periodic, } \quad \int_{Q} \psi_{p e r}^{2}=1,
\end{array}\right.
$$

and $\psi_{\text {per }} \in L^{\infty}\left(\mathbf{R}^{3}\right)$. On the other hand, we set $\alpha=\left(\varepsilon^{\prime}-\mu\right) /(1-\theta)$ and we define the function

$$
\psi_{1}(x)=\frac{\mathrm{e}^{-\sqrt{\alpha}|x|}}{|x|}
$$

It satisfies, for any radius $R>0$, and thus in particular for the $R$ appearing in (129),

$$
\left\{\begin{array}{l}
-\Delta \psi_{1}+\alpha \psi_{1} \geqslant 0, \quad \text { on } B_{R}^{c} \\
\psi_{1}>0
\end{array}\right.
$$

We are now going to show that the function

$$
\psi=\psi_{1}^{(1-\theta)} \psi_{p e r}^{\theta}
$$

is a supersolution to the equation in (129), i.e.

$$
-\Delta \psi+W \psi+\varepsilon^{\prime} \psi \geqslant 0, \quad \text { on } B_{R}^{c} .
$$

Indeed, the point is to remark that by convexity

$$
-\Delta \psi=-\Delta\left(\psi_{1}^{\theta} \psi_{p e r}^{(1-\theta)}\right) \geqslant-(1-\theta) \frac{\psi}{\psi_{1}} \Delta \psi_{1}-\theta \frac{\psi}{\psi_{p e r}} \Delta \psi_{p e r} .
$$

Therefore,

$$
\begin{aligned}
-\Delta \psi+W \psi+\varepsilon^{\prime} \psi \geqslant & \frac{\psi}{\psi_{1}}\left[-(1-\theta) \Delta \psi_{1}+\varepsilon^{\prime} \psi_{1}-\mu \psi_{1}\right] \\
& +\frac{\psi}{\psi_{p e r}}\left[-\theta \Delta \psi_{p e r}+W \psi_{p e r}+\mu \psi_{p e r}\right] \\
\geqslant & 0,
\end{aligned}
$$

in view of (131) and (130). The function $\psi$ is therefore a supersolution.

Because of (128), we know that a fortiori the first eigenvalue of the operator $-\Delta+W+\varepsilon^{\prime}$ on any bounded domain with homogeneous Dirichlet boundary conditions is also strictly positive. Therefore, this operator satisfies the maximum principle on any bounded domain. We now choose a large enough constant $C$ such that $\varphi_{1} \leqslant C \psi$ on $\partial B_{R}$. By a standard argument that we leave to the reader, we obtain $\varphi_{1} \leqslant C \psi$ on $B_{R}^{c}$, and, as $\psi$ has an exponential decay, this yields the expected behaviour of $\varphi_{1}$ at infinity.

As usual in the concentration compactness approach, the information that we now have at our disposal on the exponential decay of $\varphi_{i}$ will now be used to evaluate in a precise way the behaviour of the energy. 
For this purpose, we consider the following sequence

$$
\bar{\varphi}_{n}(x)=\sqrt{\frac{\rho(x)}{\sum_{k \in \mathbf{Z}^{3}}\left(\varphi_{1}(x+k)+\varphi_{2}(x+k+n e)\right)^{2}}}\left(\varphi_{1}(x)+\varphi_{2}(x+n e)\right),
$$

where $e$ denotes the unitary vector $(1,0,0) \in \mathbf{R}^{3}$.

The function $\bar{\varphi}_{n}$ has been designed in such a way that

$$
\sum_{k \in \mathbf{Z}^{3}} \bar{\varphi}_{n}^{2}(\cdot+k)=\rho .
$$

In addition, because of the exponential decay of $\varphi_{1}$ and $\varphi_{2}$ at infinity, we have, for some $\delta>0$,

$$
\sum_{k \in \mathbf{Z}^{3}}\left(\varphi_{1}(x+k)+\varphi_{2}(x+k+n e)\right)^{2}=\rho_{1}+\rho_{2}+\mathrm{O}\left(\mathrm{e}^{-\delta n}\right)=\rho+\mathrm{O}\left(\mathrm{e}^{-\delta n}\right),
$$

uniformly on $\mathbf{R}^{3}$. Let us now evaluate the energy of $\bar{\varphi}_{n}$. Because of (133), we must have for all $n$

$$
I_{\rho} \leqslant \int_{\mathbf{R}^{3}}\left|\nabla \bar{\varphi}_{n}\right|^{2}-\frac{1}{2} D\left(\bar{\varphi}_{n}^{2}, \bar{\varphi}_{n}^{2}\right) .
$$

On the other hand, using (134) and again the exponential decay of $\varphi_{1}$ and $\varphi_{2}$ at infinity, we may compute

$$
\begin{aligned}
\int_{\mathbf{R}^{3}}\left|\nabla \bar{\varphi}_{n}\right|^{2}-\frac{1}{2} D\left(\bar{\varphi}_{n}^{2}, \bar{\varphi}_{n}^{2}\right)= & \int_{\mathbf{R}^{3}}\left|\nabla \varphi_{1}\right|^{2}-\frac{1}{2} D\left(\varphi_{1}^{2}, \varphi_{1}^{2}\right)+\int_{\mathbf{R}^{3}}\left|\nabla \varphi_{2}\right|^{2}-\frac{1}{2} D\left(\varphi_{2}^{2}, \varphi_{2}^{2}\right) \\
& -D\left(\varphi_{1}^{2}, \varphi_{2}^{2}(\cdot+n e)\right)+\mathrm{O}\left(\mathrm{e}^{-\delta n}\right) .
\end{aligned}
$$

Therefore, in view of (115), we have

$$
\begin{aligned}
\int_{\mathbf{R}^{3}}\left|\nabla \bar{\varphi}_{n}\right|^{2}-\frac{1}{2} D\left(\bar{\varphi}_{n}^{2}, \bar{\varphi}_{n}^{2}\right) & =I_{\rho_{1}}+I_{\rho_{2}}-D\left(\varphi_{1}^{2}, \varphi_{2}^{2}(\cdot+n e)\right)+\mathrm{O}\left(\mathrm{e}^{-\delta n}\right) \\
& =I_{\rho_{1}}+I_{\rho_{2}}-\frac{1}{n} \int \varphi_{1}^{2} \int \varphi_{2}^{2}+\mathrm{o}\left(\frac{1}{n}\right),
\end{aligned}
$$

which, for $n$ large enough and along with (135), establishes (118) and contradicts (113).

Step 4: Conclusion.

In the preceding step, we have assumed for clarity that the dichotomy involves two pieces that are compact (the second one up to a translation). A case that we have on purpose omitted is the case when one part of the original sequence is compact, while the other one, vanishes. In that case, $\varphi_{2} \equiv 0$ in the preceding proof and therefore (136) does not allow to conclude. One thus has to use another strategy. What we are going to show is that the work we have made in Step 2 to exclude the case of vanishing allows to conclude also in that case. Assume that the original minimizing sequence $\varphi_{n}$ splits into $\varphi_{1}>0$ (the one that is defined at the beginning of Step 3) and $\varphi_{2, n}$ such 
that the support of $\varphi_{2, n}$ goes to infinity (in the sense made precise by the dichotomy assertion), and that moreover vanishes in the sense of Step 2 . That is, for any sequence $\left(y_{n}\right)$ of $\mathbf{R}^{3}, \varphi_{2, n}\left(\cdot+y_{n}\right)=\left(\varphi_{n}-\varphi_{1}\right)\left(\cdot+y_{n}\right)$ converges to 0 weakly in $H^{1}\left(\mathbf{R}^{3}\right)$, and, actually, $\varphi_{2, n}$ converges to 0 strongly in $L^{p}\left(\mathbf{R}^{3}\right)$, for every $2<p<6$ (see [34]). Let $\rho_{2, n}=\sum_{k \in \mathbf{Z}^{3}} \varphi_{2, n}^{2}(\cdot+k)$. Then, $\rho_{2, n}$ is non-negative, $Q$-periodic, and, since the sequence $\sqrt{\rho_{2, n}}$ is bounded in $H_{p e r}^{1}(Q)$, it converges (maybe up to the extraction of a subsequence) to $\sqrt{\rho_{2}}$, with $\rho_{2}$ non-negative and $Q$-periodic, weakly in $H_{p e r}^{1}(Q)$, and strongly in $L_{l o c}^{p}\left(\mathbf{R}^{3}\right)$, for every $1 \leqslant p<3$, thanks to Rellich's theorem. We now check that, necessarily, $\rho_{2}=\rho-\sum_{k \in \mathbf{Z}^{3}} \varphi_{1}^{2}(\cdot+k)$ (and therefore the entire sequence $\rho_{2, n}$ converges, not only a subsequence). Indeed, by observing that

$$
\rho_{2, n}=\rho_{n}-\sum_{k \in \mathbf{Z}^{3}} \varphi_{1}^{2}(\cdot+k)-2 \sum_{k \in \mathbf{Z}^{3}} \varphi_{1}(\cdot+k) \varphi_{2, n}(\cdot+k),
$$

we get

$$
\sum_{k \in \mathbf{Z}^{3}} \int_{Q} \varphi_{1}(x+k)\left|\varphi_{2, n}(x+k)\right| \mathrm{d} x=\int_{\mathbf{R}^{3}} \varphi_{1}(x)\left|\varphi_{2, n}(x)\right| \mathrm{d} x .
$$

We then easily conclude that $\sum_{k \in \mathbf{Z}^{3}} \varphi_{1}(\cdot+k) \varphi_{2, n}(\cdot+k)$ converges to 0 in $L_{l o c}^{1}\left(\mathbf{R}^{3}\right)$, using for example the two facts that $\varphi_{2, n}$ converges to 0 in $L^{4}\left(\mathbf{R}^{3}\right)$ and that $\varphi_{1}$ lies in the corresponding dual space, that is $L^{4 / 3}\left(\mathbf{R}^{3}\right)$. Therefore, we deduce, by convexity, that

$$
\begin{aligned}
\liminf _{n \rightarrow+\infty}\left|\nabla \varphi_{n}\right|^{2} & =\int_{\mathbf{R}^{3}}\left|\nabla \varphi_{1}\right|^{2}+\liminf _{n \rightarrow+\infty} \int_{\mathbf{R}^{3}}\left|\nabla \varphi_{2, n}\right|^{2} \\
& \geqslant \int_{\mathbf{R}^{3}}\left|\nabla \varphi_{1}\right|^{2}+\liminf _{n \rightarrow+\infty} \int_{Q}\left|\nabla \sqrt{\rho_{2, n}}\right|^{2} \\
& \geqslant \int_{\mathbf{R}^{3}}\left|\nabla \varphi_{1}\right|^{2}+\int_{Q}\left|\nabla \sqrt{\rho_{2}}\right|^{2} .
\end{aligned}
$$

Moreover, since $\varphi_{n}^{2}$ converges to $\varphi_{1}^{2}$ strongly in $L^{p}\left(\mathbf{R}^{3}\right)$, for every $1<p<3, \varphi_{n}^{2} \star \frac{1}{|x|}$ converges to $\varphi_{1}^{2} \star \frac{1}{|x|}$ strongly in $L^{q}\left(\mathbf{R}^{3}\right)$, for every $3<q<+\infty$. Hence, we have

$$
\lim _{n \rightarrow+\infty}-\frac{1}{2} D\left(\varphi_{n}^{2}, \varphi_{n}^{2}\right)=-\frac{1}{2} D\left(\varphi_{1}^{2}, \varphi_{1}^{2}\right)
$$

It follows that

$$
\begin{aligned}
I_{p e r}^{m, H} \geqslant & \int_{\mathbf{R}^{3}}\left|\nabla \varphi_{1}\right|^{2}+\int_{Q}\left|\nabla \sqrt{\rho_{2}}\right|^{2}-\frac{1}{2} D\left(\varphi_{1}^{2}, \varphi_{1}^{2}\right) \\
& +\frac{1}{2} D_{G}(\rho-m, \rho-m)-\frac{1}{2} D_{G}(m, m) .
\end{aligned}
$$


In order to reach a contradiction, it therefore suffices to exhibit some $\varphi_{2} \in \mathcal{D}\left(\mathbf{R}^{3}\right), \varphi_{2} \geqslant 0$, that satisfies $\sum_{k \in \mathbf{Z}^{3}} \varphi_{2}^{2}(\cdot+k)=\rho_{2}$ and

$$
\int_{\mathbf{R}^{3}}\left|\nabla \varphi_{2}\right|^{2}-\frac{1}{2} D\left(\varphi_{2}^{2}, \varphi_{2}^{2}\right)<\int_{Q}\left|\nabla \sqrt{\rho_{2}}\right|^{2},
$$

and this is proven exactly like in Step 2. Indeed, we now check that

$$
\begin{aligned}
I_{p e r}^{m, H} \leqslant & \int_{\mathbf{R}^{3}}\left|\nabla \varphi_{1}\right|^{2}+\int_{\mathbf{R}^{3}}\left|\nabla \varphi_{2}\right|^{2}-\frac{1}{2} D\left(\varphi_{1}^{2}, \varphi_{1}^{2}\right)-\frac{1}{2} D\left(\varphi_{2}^{2}, \varphi_{2}^{2}\right) \\
& +\frac{1}{2} D_{G}(\rho-m, \rho-m)-\frac{1}{2} D_{G}(m, m),
\end{aligned}
$$

in the following way. We set $\varphi_{2}^{(n)}=\varphi_{2}\left(\cdot+n e_{1}\right)$, and we consider $\tilde{\varphi}_{n}=\left(\varphi_{1}+\varphi_{2}^{(n)}\right) \| \varphi_{1}+$ $\varphi_{2}^{(n)} \|_{L^{2}\left(\mathbf{R}^{3}\right)}^{-1}$ as a test function for $I_{p e r}^{m, H}$. Then, $E_{p e r}^{m, H}\left(\tilde{\varphi}_{n}\right)=E_{p e r}^{m, H}\left(\varphi_{1}+\varphi_{2}^{(n)}\right)+\mathrm{o}(1)$, as $n$ goes to infinity, for $\left\|\tilde{\varphi}_{n}-\left(\varphi_{1}+\varphi_{2}^{(n)}\right)\right\|_{H^{1}\left(\mathbf{R}^{3}\right)}$ goes to 0 . Moreover, using the fact that $\varphi_{2}^{(n)}$ converges to 0 weakly in $H^{1}\left(\mathbf{R}^{3}\right)$, it is easily proved that $\varphi_{1} \varphi_{2}^{(n)}$ converges to 0 strongly in $L^{p}\left(\mathbf{R}^{3}\right)$, for every $1 \leqslant p \leqslant 3$. We then check without difficulty, with the help of arguments detailed before, that

$$
\lim _{n \rightarrow+\infty} D\left(\left(\varphi_{1}+\varphi_{2}^{(n)}\right)^{2},\left(\varphi_{1}+\varphi_{2}^{(n)}\right)^{2}\right)=D\left(\varphi_{1}^{2}, \varphi_{1}^{2}\right)+D\left(\varphi_{2}^{2}, \varphi_{2}^{2}\right),
$$

and that $\sum_{k \in \mathbf{Z}^{3}}\left(\varphi_{1}+\varphi_{2}^{(n)}\right)^{2}(\cdot+k)$ converges to $\rho_{1}+\rho_{2}=\rho$ in $L_{l o c}^{p}\left(\mathbf{R}^{3}\right)$, for every $1 \leqslant p<3$. Using (138), we finally obtain

$$
\begin{aligned}
I_{p e r}^{m, H} \leqslant & \limsup _{n \rightarrow \infty} E_{p e r}^{m, H}\left(\tilde{\varphi}_{n}\right) \\
= & \int_{\mathbf{R}^{3}}\left|\nabla \varphi_{1}\right|^{2}+\int_{\mathbf{R}^{3}}\left|\nabla \varphi_{2}\right|^{2}-\frac{1}{2} D\left(\varphi_{1}^{2}, \varphi_{1}^{2}\right)-\frac{1}{2} D\left(\varphi_{2}^{2}, \varphi_{2}^{2}\right) \\
& +\frac{1}{2} D_{G}(\rho-m, \rho-m)-\frac{1}{2} D_{G}(m, m) \\
< & \int_{\mathbf{R}^{3}}\left|\nabla \varphi_{1}\right|^{2}+\int_{Q}\left|\nabla \sqrt{\rho_{2}}\right|^{2}-\frac{1}{2} D\left(\varphi_{1}^{2}, \varphi_{1}^{2}\right) \\
& +\frac{1}{2} D_{G}(\rho-m, \rho-m)-\frac{1}{2} D_{G}(m, m),
\end{aligned}
$$

and this yields the desired contradiction with (137).

Since both cases of vanishing and dichotomy have been ruled out, we thus are in the case when the sequence $\varphi_{n}$ is compact in $L^{2}\left(\mathbf{R}^{3}\right)$. This concludes the proof of the theorem.

The purpose of the following subsection is to compare from above the upper limit of the energy per unit volume in the Hartree setting by the periodic Hartree model, under symmetries assumptions which are made precise in Proposition 2.1. 


\subsection{Upper limit of the energy per unit volume}

This section is devoted to the proof of the following proposition which was announced in Section 2, and which is recalled here for convenience.

Proposition 2.1. - We assume that the Van Hove sequence $\Lambda$ satisfies (41). We assume here that the unit cell $Q$ is a cube and that there exists a minimizer $\varphi_{\text {per }} \in H^{1}\left(\mathbf{R}^{3}\right)$ of $I_{p e r}^{H}$ which shares the symmetries of the unit cube. Then,

$$
\limsup _{\Lambda \rightarrow \infty} \frac{I_{\Lambda}^{H}}{|\Lambda|} \leqslant I_{p e r}^{H}+\frac{M}{2},
$$

where $I_{\text {per }}^{H}$ is defined by (30)-(31).

Remark 4.1.-

(1) The same result holds in the case of smeared nuclei, if we assume moreover that $m$ shares the symmetries of the cube $Q$, and define $M$ according to (40). However, we shall provide a proof only in the case of point nuclei, the case of smeared nuclei being even easier to deal with.

(2) In the $\mathrm{H}$ setting, since we do not know whether the minimizing $\varphi$ is unique (up to a translation), we are not able to prove that $\varphi$ shares the symmetries of the cube; this is the reason why this is an assumption in the statement of the above proposition. However, this assumption is very natural from the physical point of view.

An easy by-product of the above result is the following. The argument which rules out the vanishing case in the proof of the existence of a minimizer of $I_{p e r}^{H}$, by the concentration-compactness method, yields in particular that, given a $Q$-periodic function $\rho \geqslant 0$ such that $\sqrt{\rho} \in H_{\text {unif }}^{1}\left(\mathbf{R}^{3}\right)$ and $\int_{Q} \rho=1$, we may find a function $\varphi \in H^{1}\left(\mathbf{R}^{3}\right)$, with $\sum_{k \in \mathbf{Z}^{3}} \varphi^{2}(\cdot-k)=\rho$ (and, thus $\left.\int_{\mathbf{R}^{3}} \varphi^{2}=1\right)$, such that

$$
\int_{\mathbf{R}^{3}}|\nabla \varphi|^{2}-\frac{1}{2} D\left(\varphi^{2}, \varphi^{2}\right)<\int_{Q}|\nabla \sqrt{\rho}|^{2}
$$

(see (97) and (98) in Section 4.3). Therefore, applying this result to the density $\rho$ which minimizes $I_{p e r}^{R H}$, we make use of the corresponding function $\varphi$ as a test-function for $I_{p e r}^{H}$, to obtain

$$
\limsup _{\Lambda \rightarrow \infty} \frac{I_{\Lambda}^{H}}{|\Lambda|} \leqslant I_{p e r}^{H}+\frac{M}{2} \leqslant E_{p e r}^{H}(\varphi)+\frac{M}{2}<E_{p e r}^{R H}(\rho)+\frac{M}{2}=I_{p e r}^{R H}+\frac{M}{2} .
$$

Thus, while passing to the thermodynamic limit in the energy per unit volume, the Hartree model does not degenerate to the model $I_{p e r}^{R H}$, which would be the case if the sum of the self-interaction of the electrons was negligible with respect to $|\Lambda|$.

The rest of this section is now devoted to the

Proof of Proposition 2.1. - Let us denote by $\varphi_{\text {per }}$ a minimizer of the periodic $\mathrm{H}$ problem which shares the symmetries of the unit cube. According to the definitions (19), 
(20) and (21) of the Hartree problem, the $|\Lambda|$ functions $\varphi_{\text {per }}(\cdot-k)$, for $k$ describing $\Lambda$, are test-functions for $I_{\Lambda}^{H}$, and

$$
\begin{aligned}
I_{\Lambda}^{H} & \leqslant E_{\Lambda}\left(\left\{\varphi_{p e r}(\cdot-k) ; k \in \Lambda\right\}\right) \\
& =|\Lambda|\left[\int_{\mathbf{R}^{3}}\left|\nabla \varphi_{p e r}\right|^{2}-\frac{1}{2} D\left(\varphi_{p e r}^{2}, \varphi_{p e r}^{2}\right)\right]-\int_{\mathbf{R}^{3}} V_{\Lambda} \rho_{\Lambda}+\frac{1}{2} D\left(\rho_{\Lambda}, \rho_{\Lambda}\right)+\frac{1}{2} U_{\Lambda},
\end{aligned}
$$

where $\rho_{\Lambda}=\sum_{k \in \Lambda} \varphi_{p e r}^{2}(\cdot-k)$. If we compare now with the definitions (30) and (31) of the periodic Hartree model, we observe that proving (139) amounts to proving that

$$
\begin{gathered}
\lim _{\Lambda \rightarrow \infty} \frac{1}{|\Lambda|}\left[-\int_{\mathbf{R}^{3}} V_{\Lambda} \rho_{\Lambda}+\frac{1}{2} D\left(\rho_{\Lambda}, \rho_{\Lambda}\right)+\frac{1}{2} U_{\Lambda}\right] \\
=-\int_{Q} G \rho_{p e r}+\frac{1}{2} D_{G}\left(\rho_{p e r}, \rho_{\text {per }}\right)+\frac{M}{2} .
\end{gathered}
$$

First of all, we write down equivalent expressions for the effective potential $\Phi_{\Lambda}$, with the help of the definition of $\rho_{\Lambda}$, in the following way.

$$
\Phi_{\Lambda}(x)=V_{\Lambda}-\rho_{\Lambda} \star \frac{1}{|x|}=\sum_{k \in \Lambda}\left(\frac{1}{|x-k|}-\int_{\mathbf{R}^{3}} \frac{\varphi_{p e r}(y)^{2}}{|x-k-y|} \mathrm{d} y\right) .
$$

We first make the following observation. Since $\varphi_{\text {per }}$ shares the symmetries of the unit cube, and since $\int_{\mathbf{R}^{3}} \varphi_{\text {per }}^{2}=1$, we may show the existence of a positive constant $C$ such that

$$
\left|\frac{1}{|x|}-\int_{\mathbf{R}^{3}} \frac{\varphi_{p e r}^{2}(y)}{|x-y|} \mathrm{d} y\right| \leqslant \frac{C}{|x|^{4}},
$$

for almost every $x$ in $\mathbf{R}^{3}$ (see [32] and [11]). Let us emphasize the fact that the symmetry assumption on $\varphi_{p e r}$ is crucial for this bound to hold (see more details in [11]). We now introduce

$$
\Phi_{p e r}(x)=\sum_{k \in \mathbf{Z}^{3}}\left(\frac{1}{|x-k|}-\int_{\mathbf{R}^{3}} \frac{\varphi_{p e r}(y)^{2}}{|x-k-y|} \mathrm{d} y\right) .
$$

Because of (141), the series arising in the right-hand side of the definition of $\Phi_{p e r}$ is absolutely convergent on $\mathbf{R}^{3}$ and even uniformly convergent on the compact subsets of $\mathbf{R}^{3} \backslash \mathbf{Z}^{3}$. Moreover, since $\Phi_{\text {per }}$ is clearly $Q$-periodic, and satisfies

$$
-\Delta \Phi_{p e r}=4 \pi\left[\sum_{k \in \mathbf{Z}^{3}} \delta_{k}-\rho_{p e r}\right]
$$

(at least in the sense of distributions), we deduce that, there exists a constant $d$, such that

$$
\Phi_{p e r}=G-G \star_{Q} \rho_{p e r}+d,
$$


for $G-G \star_{Q} \rho_{\text {per }}$ is another $Q$-periodic solution to (142). Then, we prove like in [11] or [32], that

$$
\left\|\Phi_{\Lambda}\right\|_{L^{p}\left(\mathbf{R}^{3}\right)} \leqslant C|\Lambda|^{1 / p}, \quad \text { for all } 1 \leqslant p<3,
$$

where, otherwise specified, $C$ denotes here and below various positive constants that are independent of $\Lambda$,

$$
\frac{1}{|\Lambda|} \int_{\Gamma(\Lambda)^{c}}\left|\Phi_{\Lambda}\right|^{p} \rightarrow 0, \quad \text { for all } 1 \leqslant p<+\infty
$$

and

$$
\frac{1}{|\Lambda|} \int_{\Gamma(\Lambda)}\left|\Phi_{\Lambda}-\Phi_{p e r}\right|^{p} \rightarrow 0, \quad \text { for all } 1 \leqslant p<+\infty,
$$

as $\Lambda$ goes to infinity. From the proof of the existence of a minimizer for $I_{p e r}^{H}$, we know that there exist positive constants $C$ and $\mu$ such that

$$
0 \leqslant \varphi_{p e r}(x) \leqslant C \exp (-\mu|x|), \quad \text { a.e. on } \mathbf{R}^{3},
$$

and then, the analogous bounds and convergence results are also easily proved for $\rho_{\Lambda}$; that is

$$
\begin{aligned}
& \left\|\rho_{\Lambda}\right\|_{L^{p}\left(\mathbf{R}^{3}\right)} \leqslant C|\Lambda|^{1 / p}, \quad \text { for all } 1 \leqslant p \leqslant+\infty \\
& \frac{1}{|\Lambda|} \int_{\Gamma(\Lambda)^{c}}\left|\rho_{\Lambda}\right|^{p} \rightarrow 0, \quad \text { for all } 1 \leqslant p<+\infty
\end{aligned}
$$

and

$$
\frac{1}{|\Lambda|} \int_{\Gamma(\Lambda)}\left|\rho_{\Lambda}-\rho_{p e r}\right|^{p} \rightarrow 0, \quad \text { for all } 1 \leqslant p<+\infty,
$$

as $\Lambda$ goes to infinity.

We may now turn to the proof of (140). First, we check that

$$
\lim _{\Lambda \rightarrow \infty} \frac{1}{|\Lambda|}\left[-\frac{1}{2} \int_{\mathbf{R}^{3}} V_{\Lambda} \rho_{\Lambda}+\frac{1}{2} U_{\Lambda}\right]=-\frac{1}{2} \int_{Q} G \rho_{p e r}+\frac{M}{2}+\frac{d}{2} .
$$

Indeed, we have

$$
\begin{aligned}
\frac{1}{|\Lambda|}\left[-\frac{1}{2} \int_{\mathbf{R}^{3}} V_{\Lambda} \rho_{\Lambda}+\frac{1}{2} U_{\Lambda}\right] & =\frac{1}{2|\Lambda|} \sum_{k \in \Lambda} \lim _{x \rightarrow k}\left(\Phi_{\Lambda}(x)-\frac{1}{|x-k|}\right) \\
& =\frac{1}{2|\Lambda|} \sum_{k \in \Lambda} \lim _{x \rightarrow 0}\left(\Phi_{\Lambda}(x+k)-\frac{1}{|x|}\right) \\
& =\frac{1}{2} \lim _{x \rightarrow 0}\left(\tilde{\Phi}_{\Lambda}(x)-\frac{1}{|x|}\right),
\end{aligned}
$$


and it is shown in [11] that $\tilde{\Phi}_{\Lambda}$ converges to $\Phi_{\text {per }}$ uniformly on the compact subsets of $\mathbf{R}^{3}$. Thus, thanks to the definition of $\Phi_{\text {per }}$, we get

$$
\lim _{\Lambda \rightarrow \infty} \frac{1}{2} \lim _{x \rightarrow 0}\left(\tilde{\Phi}_{\Lambda}(x)-\frac{1}{|x|}\right)=\frac{1}{2} \lim _{x \rightarrow 0}\left(\Phi_{p e r}(x)-\frac{1}{|x|}\right)=-\frac{1}{2} \int_{Q} G \rho_{p e r}+\frac{M}{2}+\frac{d}{2},
$$

which gives (149).

Secondly, we establish that

$$
\begin{aligned}
& \lim _{\Lambda \rightarrow \infty} \frac{1}{|\Lambda|}\left[-\frac{1}{2} \int_{\mathbf{R}^{3}} V_{\Lambda} \rho_{\Lambda}+\frac{1}{2} D\left(\rho_{\Lambda}, \rho_{\Lambda}\right)\right] \\
& =-\frac{1}{2} \int_{Q} G \rho_{p e r}+\frac{1}{2} D_{G}\left(\rho_{p e r}, \rho_{p e r}\right)-\frac{d}{2},
\end{aligned}
$$

by remarking that

$$
\begin{aligned}
& \frac{1}{|\Lambda|}\left[-\frac{1}{2} \int_{\mathbf{R}^{3}} V_{\Lambda} \rho_{\Lambda}+\frac{1}{2} D\left(\rho_{\Lambda}, \rho_{\Lambda}\right)\right] \\
& =-\frac{1}{2|\Lambda|} \int_{\mathbf{R}^{3}} \Phi_{\Lambda} \rho_{\Lambda}=-\frac{1}{2|\Lambda|} \int_{\Gamma(\Lambda)} \Phi_{\Lambda} \rho_{\Lambda}+\mathrm{o}(1) \\
& =-\frac{1}{2|\Lambda|} \int_{\Gamma(\Lambda)} \Phi_{p e r} \rho_{p e r}+\mathrm{o}(1)=-\frac{1}{2} \int_{Q} \Phi_{p e r} \rho_{p e r}+\mathrm{o}(1) \\
& =-\frac{1}{2} \int_{Q} G \rho_{p e r}+\frac{1}{2} D_{G}\left(\rho_{p e r}, \rho_{p e r}\right)-\frac{d}{2}+\mathrm{o}(1),
\end{aligned}
$$

as $\Lambda$ goes to infinity. This string of equalities is straightforwardly verified with the help of (143), (144), (145), (146), (147), and (148). The proof of (140) (and thus of Proposition 2.1) follows gathering together (149) and (150).

\subsection{Some final comments on the Hartree type models}

Theorem 2.2 deserves some comments, that we list in this paragraph.

First of all, let us notice that Theorem 2.2 provides an existence result of a normalized $\left(\int_{\mathbf{R}^{3}} \varphi^{2}=1\right)$ solution to the associated Euler-Lagrange equation, namely

$$
-\Delta \varphi-\left(\varphi^{2} \star \frac{1}{|x|}\right) \varphi+\left(G \star_{Q}(\rho-m)\right) \varphi+\varepsilon \varphi=0 .
$$

Existence (and bifurcation) results for this type of nonlinear equation (Choquard-Pekar equation) have already been obtained by B. Buffoni, L. Jeanjean, Ch. Stuart et al. [7, 8,20-22,50], but as far as we know, the existence of a normalized solution was still an open question. Theorem 2.2 settles this question. 
Secondly, it is usual in Solid State Physics to consider Hartree-type equations of the more general form

$$
-\Delta \varphi+F(\varphi) \varphi+W \varphi+\varepsilon \varphi=0,
$$

where $F(\varphi) \varphi$ is some local correction to the mean-field potential $W$. For example, in (151), $W=G \star_{Q}(\rho-m)$ is the electrostatic periodic potential created by the periodic lattice of nuclei and by the electronic density $\rho$, while the more usual term $-\left(\varphi^{2} \star \frac{1}{|x|}\right) \varphi$ takes into account the self-interaction of each electron with itself. Therefore, it seems to us that the Hartree equation (151) we have obtained is likely to be not so far from equations used by Solid State physicists.

Thirdly, we must confess it may seem surprising that such an apparently easy minimization problem, set on the unit cell $Q$ with periodic boundary conditions, leads to such a complicated proof. However, we have not been able (so far) to simplify the above proof. In some sense, one can find some relationship between our strategy of proof and ideas developed by $\mathrm{O}$. Lopes in $[37,38,36]$ for some translation invariant problems of a similar type.

Finally, let us emphasize again that we do not know of any rigorous proof of the fact that this periodic minimization problem is indeed obtained in the thermodynamic limit for an arbitrary Hartree type model. It must be clear to the reader that the only case when we are able to conclude (Proposition 4.1) is very particular. Nevertheless, we believe it has some kind of generality. At least, we hope that the present suggestion for a periodic Hartree model will stimulate further research.

\section{Extensions and perspectives}

We list in this last section a few comments on the above results, and indicate some possible extensions of our work.

So far, we have assumed that the periodic lattice that is covered in the limit by the sequence $\Lambda$ is $\mathbf{Z}^{3}$, and thus that the periodic cell $Q$ is a cube of unit size. The first basic observation to make is that our whole work goes through mutatis mutandis if we replace the cube of unit size by a cube of size $R$. Slight modifications must be made in the definition of the potential $G$ in particular, and we refer the reader to [11] for such modifications.

Replacing the cube by another shape of unit cell is another story. As we have mentioned above, Theorems 2.1 and 2.2 still hold. So does Proposition 4.1. On the contrary, our strategy of proof for Proposition 2.1 depends upon the shape of the cell. It is an open (but rather technical) question to extend this result to other shapes of cells.

Likewise, we have mentioned above that the assumption (41) is a technical assumption required only for the proof of Proposition 2.1. We recall we believe it can be skipped, but we do not know how.

Apart from these side issues, the main open problem to tackle is the proof of the thermodynamic limit for the energy per unit volume in the Hartree model. As far as this question is concerned, a lot remains to be done. 
Even in some simplified framework, trying to understand Hartree type models for quasicrystals would also be of interest. Our study [11] and references [1,3,6,45] could constitute a starting point.

Let us also mention that the periodic problems we have defined in this work can be treated numerically, and we indeed intend to treat them numerically. Numerical experiment might in particular give some insight into the mathematical nature of these models and help oneself to make up his mind on some of the questions mentioned above.

We finally recall from the introduction that the same issues on the Hartree-Fock model (and some of its simplified form) are studied by the authors in [13].

\section{REFERENCES}

[1] Amerio L., Prouse G., Almost Periodic Functions and Functional Equations, Van Nostrand Reinhold, 1971.

[2] Ashcroft N.W., Mermin N.D., Solid-state Physics, Saunders College Publishing, 1976.

[3] Axel F., Gratias D. (Eds.), Beyond Quasicrystals, Centre de Physique Les Houches, Les Editions de Physique, Springer, 1995.

[4] Balian R., From Microphysics to Macrophysics; Methods and Applications of Statistical Physics, I \& II, Springer-Verlag, 1991.

[5] Benguria R., Brézis H., Lieb E.H., The Thomas-Fermi-von Weizsäcker theory of atoms and molecules, Comm. Math. Phys. 79 (1981) 167-180.

[6] Bohr H., Almost Periodic Functions, Chelsea, 1947.

[7] Buffoni B., Jeanjean L., Stuart C.A., Existence of a non-trivial solution to a strongly indefinite semilinear equation, Proc. Amer. Math. Soc. 119 (1993) 179-186.

[8] Buffoni B., Jeanjean L., Minimax characterization of solutions for a semi-linear elliptic equation with lack of compactness, Ann. Inst. Henri Poincaré, Anal. non lin. 10 (4) (1993) 377-404.

[9] Callaway J., Quantum Theory of the Solid State, Academic Press, 1974.

[10] Catto I., Le Bris C., Lions P.-L., Limite thermodynamique pour des modèles de type Thomas-Fermi, C. R. Acad. Sci. Paris, Série I 322 (1996) 357-364.

[11] Catto I., Le Bris C., Lions P.-L., Mathematical Theory of Thermodynamic Limits: ThomasFermi Type Models, Oxford University Press, 1998.

[12] Catto I., Le Bris C., Lions P.-L., Sur la limite thermodynamique pour des modèles de type Hartree et Hartree-Fock, C. R. Acad. Sci. Paris, Série I 327 (1998) 259-266.

[13] Catto I., Le Bris C., Lions P.-L., On the thermodynamic limit for Hartree-Fock type models, Ann. Inst. Henri Poincaré, to appear.

[14] Dreizler R.M., Gross E.K.U., Density Functional Theory, Springer-Verlag, 1990.

[15] Eastham M.S.P., The Spectral Theory of Periodic Differential Equations, Scottish Acad. Press, Edinburgh, 1973.

[16] Ekeland I., Nonconvex minimization problems, Bull. Amer. Math. Soc. 1 (3) (1979) 443474.

[17] Fefferman C., The thermodynamic limit for a crystal, Comm. Math. Phys. 98 (1985) 289311.

[18] Gregg J.N. Jr., The existence of the thermodynamic limit in Coulomb-like systems, Comm. Math. Phys. 123 (1989) 255-276.

[19] Hartree D., The wave-mechanics of an atom with a non-Coulomb central field. Part I. Theory and methods, Proc. Comb. Phil. Soc. 24 (1928) 89-132. 
[20] Heinz H.-P., Küpper T., Stuart C.A., Existence and bifurcation of solutions for nonlinear perturbations of the periodic Schrödinger equation, J. Differential Equations 100 (1992) 341-354.

[21] Jeanjean L., Solutions in the spectral gap for a nonlinear equation of Schrödinger type, J. Differential Equations 112 (1994) 53-80.

[22] Jeanjean L., Existence of solutions with prescribed norm for semilinear elliptic equations, Nonlinear Analysis TMA 28 (10) (1997) 1633-1659.

[23] Kittel C., Introduction to Solid-State Physics, 6th edn., Wiley, 1986.

[24] Lebowitz J.L., Lieb E.H., Existence of thermodynamics for real matter with Coulomb forces, Phys. Rev. Lett. 22 (13) (1969) 631-634.

[25] Lieb E.H., Lebowitz J.L., The constitution of matter: existence of thermodynamics for systems composed of electrons and nuclei, Adv. Math. 9 (1972) 316-398.

[26] Lieb E.H., Lebowitz J.L., Lectures on the thermodynamic limit for Coulomb systems, in: Lecture Notes in Physics, Vol. 20, Springer, 1973, pp. 136-161.

[27] Lieb E.H., The stability of matter, Rev. Mod. Phys. 48 (1976) 553-569.

[28] Lieb E.H., Existence and uniqueness of the minimizing solution of Choquard's nonlinear equation, Studies in Appl. Math. 57 (1977) 93-105.

[29] Lieb E.H., Thomas-Fermi and related theories of atoms and molecules, Rev. Mod. Phys. 53 (4) (1981) 603-641.

[30] Lieb E.H., The stability of matter: from atoms to stars, Bull. Amer. Math. Soc. 22 (1) (1990) $1-49$.

[31] Lieb E.H., Narnhofer H., The thermodynamic limit for Jellium, J. Stat. Phys. 12 (1975) 291-310.

[32] Lieb E.H., Simon B., The Thomas-Fermi theory of atoms, molecules and solids, Adv. Math. 23 (1977) 22-116.

[33] Lieb E.H., Simon B., The Hartree-Fock theory for Coulomb systems, Comm. Math. Phys. 53 (1977) 185-194.

[34] Lions P.-L., The concentration-compactness principle in the calculus of variations. The locally compact case, Parts 1 \& 2, Ann. Inst. H. Poincaré 1 (1984) 109-145 and 223-283.

[35] Lions P.-L., Solutions of Hartree-Fock equations for Coulomb systems, Comm. Math. Phys. 109 (1987) 33-97.

[36] Lopes O., A constrained minimization problem with integrals on the entire space, Bol. Soc. Bras. Mat., Nova Ser. 25 (1) (1994) 77-92.

[37] Lopes O., Sufficient conditions for minima of some translation invariant functionals, Differential Integral Equations 10 (2) (1997) 231-244.

[38] Lopes O., Variational problems defined by integrals on the entire space and periodic coefficients, Comm. Appl. Nonlinear Anal. 5 (2) (1998) 87-120.

[39] Madelung O., Introduction to Solid-State Theory, Solid State Sciences, Vol. 2, Springer, 1981.

[40] Pisani C., Quantum Mechanical Ab Initio Calculation of the Properties of Crystalline Materials, Lecture Notes in Chemistry, Vol. 67, Springer, 1996.

[41] Parr R.G., Yang W., Density-Functional Theory of Atoms and Molecules, Oxford University Press, Oxford, 1989.

[42] Quinn Ch.M., An Introduction to the Quantum Theory of Solids, Clarendon Press, Oxford, 1973.

[43] Reed M., Simon B., Methods of Modern Mathematical Physics, IV, Academic Press, New York, 1978.

[44] Ruelle D., Statistical Mechanics: Rigorous Results, Benjamin, New York, 1969 and Advanced Books Classics, Addison-Wesley, 1989. 
[45] Senechal M., Quasicrystals and Geometry, Cambridge University Press, 1995.

[46] Simon B., Schrödinger semi-groups, Bull. Amer. Math. Soc. 7 (3) (1982) 447-526.

[47] Slater J.C., Quantum Theory of Molecules and Solids, Mac Graw Hill, 1963.

[48] Slater J.C., Symmetry and Energy Bands in Crystals, Dover, 1972.

[49] Solovej J.P., An improvement on stability of matter in mean field theory, Proceedings of the Conference on PDEs and Mathematical Physics, Univ. of Alabama, International Press, 1994.

[50] Stuart Ch., Bifurcation into Spectral Gaps, Bulletin of the Belgian Mathematical Society, 1995.

[51] Tolman R.C., The Principles of Statistical Mechanics, Oxford University Press, 1962.

[52] Wilcox C., Theory of Bloch waves, J. Analyse Math. 33 (1978) 146-167.

[53] Ziman J., Principles of the Theory of Solids, 2nd edn., Cambridge University Press, 1972. 